\title{
EFT diagrammatica: UV roots of the CP-conserving SMEFT
}

\author{
Supratim Das Bakshi, ${ }^{a}$ Joydeep Chakrabortty, ${ }^{a}$ Suraj Prakash, ${ }^{a}$ Shakeel Ur Rahaman ${ }^{a}$ \\ and Michael Spannowsky ${ }^{b}$ \\ ${ }^{a}$ Indian Institute of Technology Kanpur, \\ Kalyanpur, Kanpur 208016, Uttar Pradesh, India \\ ${ }^{b}$ Institute for Particle Physics Phenomenology, Department of Physics, Durham University, \\ Durham DH1 3LE, U.K. \\ E-mail: sdbakshi@iitk.ac.in, joydeep@iitk.ac.in, surajprk@iitk.ac.in, \\ shakel@iitk.ac.in, michael.spannowsky@durham.ac.uk
}

Abstract: The Standard Model Effective Field Theory (SMEFT) is an established theoretical framework that parametrises the impact a UV theory has on low-energy observables. Such parametrization is achieved by studying the interactions of SM fields encapsulated within higher mass dimensional $(\geq 5)$ operators. Through judicious employment of the tools of EFTs, SMEFT has become a source of new predictions as well as a platform for conducting a coherent comparison of new physics (beyond Standard Model) scenarios. We, for the first time, are proposing a diagrammatic approach to establish selection criteria for the allowed heavy field representations corresponding to each SMEFT operator. We have elucidated the links of a chain connecting specific CP conserving dimension-6 SMEFT operators with unique sets of heavy field representations. The contact interactions representing each effective operator have been unfolded into tree- and (or) one-loop-level diagrams to reveal unique embeddings of heavy fields within them. For each case, the renormalizable vertices of a UV model serve as the building blocks for all possible unfolded diagrams. Based on this, we have laid the groundwork to construct observable-driven new physics models. This in turn also prevents us from making redundant analyses of similar models. While we have taken a predominantly minimalistic approach, we have also highlighted the necessity for non-minimal interactions for certain operators.

Keywords: Beyond Standard Model, Effective Field Theories

ArXiv EPrint: 2103.11593 


\section{Contents}

1 Introduction 1

2 Unfolding effective operators into Lorentz invariant vertices 3

3 Identifying heavy fields corresponding to dimension-6 SMEFT operators 6

$\begin{array}{lll}3.1 & \phi^{6} & 13\end{array}$

$\begin{array}{lll}3.2 & \phi^{4} \mathcal{D}^{2} & 13\end{array}$

$\begin{array}{lll}3.3 & \psi^{2} \phi^{3} & 15\end{array}$

$\begin{array}{lll}3.4 & \psi^{2} \phi^{2} \mathcal{D} & 18\end{array}$

$\begin{array}{lll}3.5 & \phi^{2} X^{2} & 18\end{array}$

$\begin{array}{lll}3.6 & \psi^{4} & 20\end{array}$

$\begin{array}{lll}3.7 & X^{3} & 31\end{array}$

4 Departure from minimality $\quad 31$

4.1 Diagrams with multiple fundamental vertices 32

4.2 Heavy-heavy mixing in the loops 32

4.3 Multi-loop diagrams 32

5 Operator driven BSM construction: validation and illustration through examples 33

5.1 Validating the diagrammatic method 33

5.2 The minimal extension of the SM as root of CP even D6 SMEFT operators 34

5.3 Role of observables on the choice of BSMs 35

$\begin{array}{lll}6 & \text { Conclusion and remarks } & 37\end{array}$

$\begin{array}{ll}\text { A The Standard model field content } & 38\end{array}$

B Products of lower dimensional $\mathrm{SU}(N)$ representations 38

\section{Introduction}

The contemporary paradigm for conducting phenomenological analyses in particle physics is based on Effective Field Theories (EFTs). EFT are a natural choice considering the proliferation of scales in subatomic physics. A popular EFT paradigm is the so-called bottom-up approach, first introduced in [1-3], which not only attempts to encapsulate the effects of unknown high energy theories but also provides useful contributions to the observables defined by the renormalizable Lagrangian. This method involves the construction of higher mass dimension operator bases, and the parameter space is defined by the Wilson 
coefficients corresponding to these operators. A number of computational tools have been developed that automate the procedure of operator construction [4-8]. Standard Model Effective Field Theory (SMEFT) is the bottom-up extension of the Standard Model which consists of 59 operators at dimension- 6 considering only a single flavour of fermions ${ }^{1}$ and brings to light several interesting features not encountered at the renormalizable level. The only downside of this approach is that it is oblivious to the exact UV roots of each of the operators and the associated phenomenology. Hence, the Wilson coefficients remain without origin and independent [16].

The other face of the coin, popularly known as the top-down approach, starts at a higher scale where one constructs the Lagrangian for a particular model and identifies certain degrees of freedom as heavy and systematically integrates them out to obtain a set of higher mass dimension operators composed entirely of the lighter fields [17-27]. To affirm the predictions of a UV theory against the SM observables, the field content of the chosen UV theory must first be brought down to the SM field content and this is where the top-down approach finds great utility. This procedure ultimately leads to various subsets of the 59 SMEFT operators, constructed via the bottom-up approach. These may or may not overlap for different UV models. Various computational packages have been developed to automate the procedure for certain cases [28-31].

Also, from the point of view of symmetries, there is no restriction on the choice of field as well as symmetry extensions of the SM that can be treated as a UV theory. The only means of eliminating candidate models is through phenomenological analyses. But, even then a large number of models still appear to be viable as they all contribute to some observable or another [32-39]. Conducting a comparative analysis of every single one of them becomes tedious and impractical, more so for non-minimal scenarios with multiple heavy fields. In such cases, we encounter a multitude of scales which implies that a cascade of EFTs may be required to determine the relations among SMEFT Wilson coefficients and BSM parameters, and a systematic procedure must be developed to address this.

It is desirable to have a more structured method of cataloguing the UV models that lead to specific SMEFT operators, which in turn can be correlated with observables. Clearly, instead of starting with a different BSM Lagrangian each time and comparing the various subsets of SMEFT each of them leads to, it is more economical if we approach this issue in a retrograde manner, where based on the observables under study we first identify the necessary operators and then attempt to enumerate the specific list of heavy fields that can generate the particular operator(s). This allows us to conduct our analysis in a minimal sense and also highlights which combinations of heavy fields may lead to redundant contributions.

This operator-driven BSM model building is what we have addressed in this work, i.e., our primary aim has been to identify the possible UV roots of each SMEFT operator when considering 1-particle-irreducible (1PI) diagrams up to one-loop-level built of interactions

\footnotetext{
${ }^{1}$ This counting does not include hermitian conjugates of the operators, nor does it include operators that violate accidental symmetries of the renormalizable Lagrangian. Taking those into account, we get 2 operators at dimension-5 [9, 10], 84 at dimension-6 [3], 30 at dimension-7 [11], 993 at dimension-8 [12, 13] and 560 operators at dimension-9 $[14,15]$ for SMEFT.
} 
involving the SM as well as heavy fields. We must emphasize that we have only considered extensions to the SM particle content by addition of spin- 0 and spin- $1 / 2$ particles, ${ }^{2}$ and the internal symmetry has been kept fixed at $\mathrm{SU}(3)_{C} \otimes \mathrm{SU}(2)_{L} \otimes \mathrm{U}(1)_{Y}$. Also, we realise that the SMEFT operators may receive radiative corrections of the SM as well as heavy fields but accounting for such corrections would require us to delve deeper into the incorporation of a renormalization prescription which is beyond the scope of the current work and such analysis will form a part of our future objectives. We have started by delineating a schematic unfolding of the set of independent operator classes of mass dimension- 6 in section 2 . The external legs of the contact operators have been judiciously segregated and internal lines have been added suitably while ensuring Lorentz invariance at each vertex to obtain a representative set of tree- and one-loop-level diagrams for each operator class and the corresponding subclasses if any. In section 3, we have first prepared an exhaustive list of heavy field representations which can appear at various renormalizable vertices when the internal symmetry of the SM is imposed in addition to Lorentz invariance. Then using these interactions as fundamental building blocks we have carefully outlined how each SMEFT operator of mass dimension-6 can be unfolded to reveal these heavy fields within tree-level diagrams with heavy propagators and one-loop-level diagrams involving pure-heavy or light-heavy-mixed loops. We must remark that the analysis here is by no means exhaustive and conforms to a notion of minimality which has been described in the paper. As a matter of fact, in section 4, we have addressed a few ways in which our set can be extended by accounting for non-minimal cases. We have also emphasized special cases of certain operators where this non-minimality is unavoidable. In section 5 , we have validated our results by focussing on a single heavy scalar representation and showing that the various SMEFT operators whose unfolding incorporates it are precisely the same as the ones obtained when the UV theory containing the particular heavy scalar is subjected to the top-down analysis [40-45]. We have also described how an extension of the SM containing a second Higgs doublet along with a scalar with a non-trivial color quantum number can be seen to account for all the operators that are significant in the context of electroweak precision observables (EWPO) and Higgs signal strength measurements [43, 44, 46-51]. In addition, we have emphasized the need for non-minimal extensions, analyses at the level of higher loops, as well as the necessity of developing novel observables to account for the remaining SMEFT operators.

\section{Unfolding effective operators into Lorentz invariant vertices}

We intend to highlight a generalizable procedure using which, provided a complete and independent set of operators, we can trace the origin of each operator from candidate UV theories containing specific heavy fields. This indeed is the opposite of conventional analyses where one starts with a UV theory, identifies certain degrees of freedom as heavy and after

\footnotetext{
${ }^{2}$ It is possible that the internal symmetry of SM may be a subset of some larger symmetry which is manifested at an as yet undetermined higher energy scale. Hence, it is fair to consider SM extensions involving heavy spin-1 particles associated with the breaking of the higher symmetry. But we have opted to exclude such scenarios in order to keep our analysis simple.
} 


\begin{tabular}{|c|c|c|c|c|c|}
\hline Symbol & Stands for & Symbol & Stands for & Symbol & Stands for \\
\hline - - - - - - - & Scalar & & Fermion & WW & $\begin{array}{c}\text { SM Gauge } \\
\text { Boson }\end{array}$ \\
\hline - - - - - - & $\begin{array}{c}\text { Light (SM) } \\
\text { scalar }(\phi)\end{array}$ & & $\begin{array}{l}\text { Light }(\mathrm{SM}) \\
\text { fermion }(\psi)\end{array}$ & & $(\cdot) \mathcal{D}^{\mu}(\cdot) \mathcal{D}_{\mu}(\cdot)$ \\
\hline - - - - - - & $\begin{array}{c}\text { Heavy } \\
\text { scalar }(\Phi)\end{array}$ & & $\begin{array}{c}\text { Heavy } \\
\text { fermion }(\Psi)\end{array}$ & & $(\cdot) \gamma^{\mu}(\cdot \cdot) \mathcal{D}_{\mu}(\cdot)$ \\
\hline (1) & $\mathcal{D}_{\mu}$ & ○ & $\gamma^{\mu}$ & $\otimes$ & $\square \equiv \mathcal{D}^{2}$ \\
\hline
\end{tabular}

Table 1. The symbols used throughout the paper and their meaning.

suitably integrating out obtains a subset of the effective Lagrangian, i.e., higher mass dimension operator basis, of a low energy theory [52-56]. The following points emphasize the salient aspects of this reverse-engineering procedure:

- The building blocks of our analysis are Feynman diagrams which in turn are constituted of certain well-defined symbols. For instance, dashed, solid, and wiggly lines indicate scalars, fermions, and gauge bosons respectively. Different colours differentiate between heavy, light, and general fields. These along with additional symbols for $\mathcal{D}_{\mu}, \gamma^{\mu}$, and their combinations have been listed in table 1 .

- At the intermediate step between these symbols and Feynman diagrams lie the vertices obtained from the renormalizable Lagrangian. ${ }^{3}$ The most general collection of vertices, which includes trilinear and quartic self-interactions of scalars and gauge bosons, Yukawa terms connecting a pair of fermions with a scalar and the kinetic terms of scalars and fermions, have been depicted in figure 1. One can see how these vertices incorporate the symbols defined in table 1.

- Though our method is generic and applicable for any effective field theory, in this work, we restrict ourselves to the effective Lagrangians of mass dimension-6. In figure 2, we have collected schematic representations of Lorentz invariant operator classes that constitute a complete and independent set at dimension-6, popularly dubbed as the Warsaw basis in the context of SMEFT [3].

- It must be noted that when Lorentz invariance is the only constraint, the contents of figure 2 are not the only possible operator classes of dimension- 6 that can be constructed out of scalars, spinors, vector bosons and their derivatives. For instance,

\footnotetext{
${ }^{3}$ It must be kept in mind that we are working in $3+1$ space-time dimensions, therefore a renormalizable Lagrangian consists of terms with mass dimension 4 and individual components, i.e., bosons, fermions, derivatives and field strength tensors have mass dimensions of $1, \frac{3}{2}, 1$ and 2 respectively.
} 

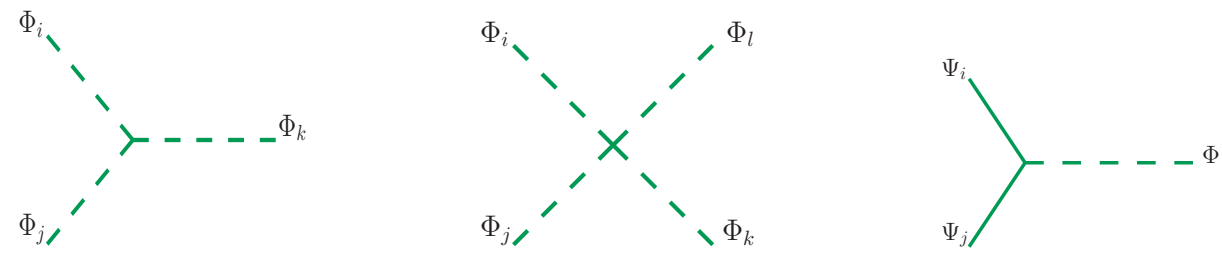

(i) $[\Phi]^{3}$.

(ii) $[\Phi]^{4}$.

(iii) $[\Psi]^{2} \Phi$.

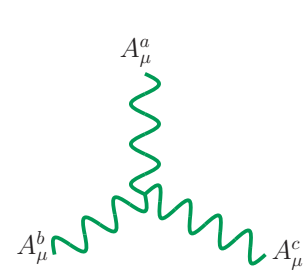

(iv) $\left[A_{\mu}\right]^{3}$.

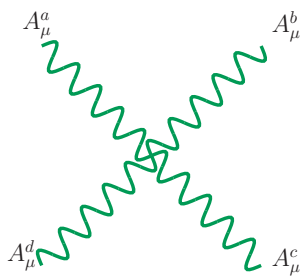

(v) $\left[A_{\mu}\right]^{4}$.

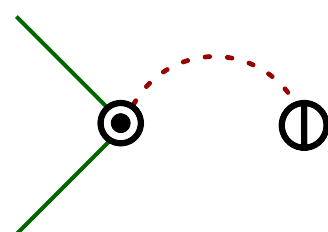

(vi) $[\Psi]^{2} \mathcal{D}$.
$\bigotimes---\Phi_{j}$

(vii) $\Phi \square \Phi$.

Figure 1. Lorentz invariant vertices obtained from terms of the renormalizable Lagrangian.

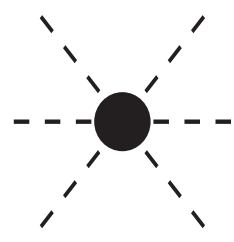

(i) $\phi^{6}$.

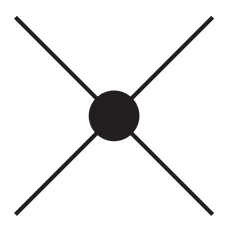

(ii) $\psi^{4}$.

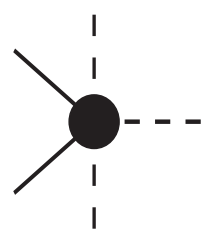

(iii) $\psi^{2} \phi^{3}$.

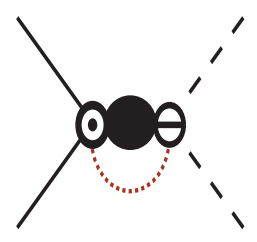

(iv) $\psi^{2} \phi^{2} \mathcal{D}$.

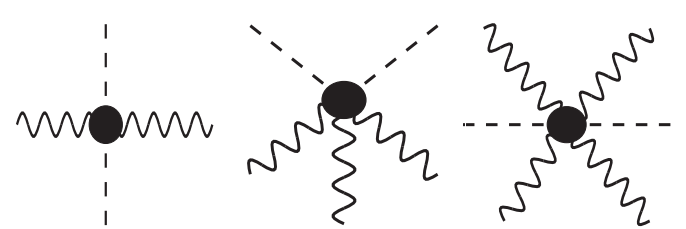

(v) $\phi^{2} X^{2}$

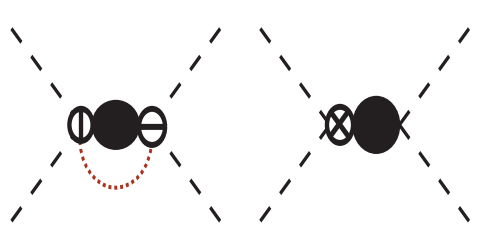

(vi) $\phi^{4} \mathcal{D}^{2}$.

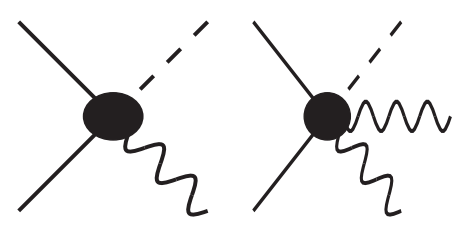

(vii) $\psi^{2} \phi X$.

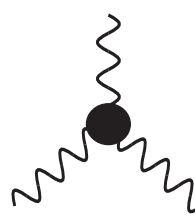

$\eta$

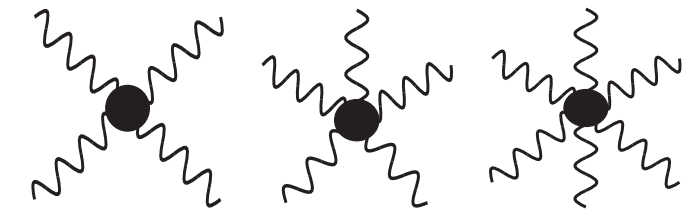

(viii) $X^{3}$.

Figure 2. Schematic representation of SMEFT operator classes at mass dimension-6 in the Warsaw basis. The dotted red line indicates the contraction of Lorentz indices as mentioned in table 1. 
classes such as $\mathcal{D}^{2} X^{2}, \psi^{2} \phi \mathcal{D}^{2}$ etc., are often taken into account to describe specific phenomenological processes but these can be expressed as linear combinations of elements of the Warsaw-like basis as shown below [3, 57]:

$$
\begin{aligned}
\underbrace{\left(\mathcal{D}_{\mu} X^{\mu \nu}\right)^{2}}_{\mathcal{D}^{2} X^{2}} & \sim c_{1} \underbrace{\left(\bar{\psi} \gamma^{\nu} \psi\right)\left(\bar{\psi} \gamma_{\nu} \psi\right)}_{\psi^{4}}+c_{2} \underbrace{\left(\bar{\psi} \gamma^{\nu} \psi\right)\left(\phi^{\dagger} i \overleftrightarrow{\mathcal{D}_{\nu}} \phi\right)}_{\psi^{2} \phi^{2} \mathcal{D}}+c_{3} \underbrace{\left(\phi^{\dagger} i \overleftrightarrow{\mathcal{D}}^{\nu} \phi\right)\left(\phi^{\dagger} i \overleftrightarrow{\mathcal{D}_{\nu}} \phi\right)}_{\phi^{4} \mathcal{D}^{2}}, \\
\underbrace{\left(\bar{\psi}_{L} \psi_{R}\right) \mathcal{D}^{2} \phi}_{\psi^{2} \phi \mathcal{D}^{2}} & \sim c_{0}^{\prime} \underbrace{\left(\bar{\psi}_{L} \psi_{R} \phi\right)}_{\text {renorm. term }}+c_{1}^{\prime} \underbrace{\left(\bar{\psi}_{L} \psi_{R} \phi\right) \phi^{\dagger} \phi}_{\psi^{2} \phi^{3}}+c_{2}^{\prime} \underbrace{\left(\bar{\psi}_{L} \psi_{R}\right)\left(\bar{\psi}_{L} \psi_{R}\right)}_{\psi^{4}} .
\end{aligned}
$$

These relations can be established through the equations of motion (EOMs) of the constituent fields:

$$
\mathcal{D}_{\mu} X^{\mu \nu}=\bar{\psi} \gamma^{\nu} \psi+\phi^{\dagger} \overleftrightarrow{\mathcal{D}}^{\nu} \phi, \quad \mathcal{D}^{2} \phi=c_{0}^{\prime} \phi+c_{1}^{\prime}\left(\phi^{\dagger} \phi\right) \phi+c_{2}^{\prime} \bar{\psi}_{1} \psi_{2}
$$

- In addition to the EOMs of the fields, operators may also be related to each other through Integration by Parts (IBPs) and certain Fierz identities. But these relations only appear when we consider individual operators, not at the level of operator class. Hence, the implications of such relations have only been discussed once we probe into the structures of specific operators.

- One can see that multiple diagrams have been drawn corresponding to the classes containing $X$ in figure 2. The reason is that each $X$ which denotes a field strength tensor can contain up to two gauge bosons within it. Thus, the multiple diagrams hint at how several effective vertices can be obtained from the same effective operator.

- The next important step is the "unfolding" of the effective operators, shown in figure 2, using the renormalizable vertices depicted in figure 1, through tree- and oneloop-level diagrams. This has been done for each class and the results have been shown in figures $3-10$.

- We must comment at this stage that the diagrams for individual classes need not be exhaustive but care has been taken to include the ones which are relevant for the most general analysis.

- Also, the unfolding at this stage has been done with only Lorentz invariance in mind. So, within the loops, we have kept open the possibility of having light as well as heavy propagators, but it must be understood that there is at least one heavy field.

\section{Identifying heavy fields corresponding to dimension-6 SMEFT op- erators}

In this section, we have substantiated the general ideas presented in the previous one using the CP even dimension-6 SMEFT operators as the backdrop, i.e., henceforth the light fields have been restricted to be Standard Model fields, described in appendix A. The sequence of steps involved can be described under two main headings: 


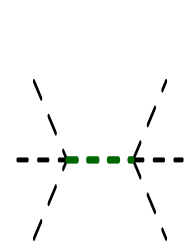

(i)

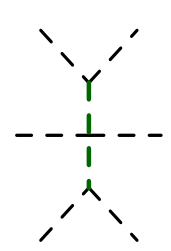

(ii)

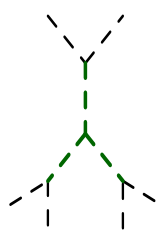

(iii)

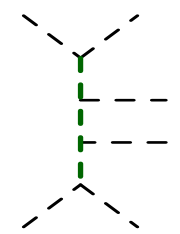

(iv)

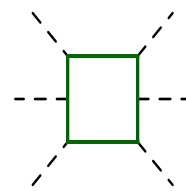

(v)

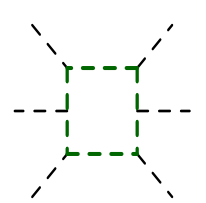

(vi)

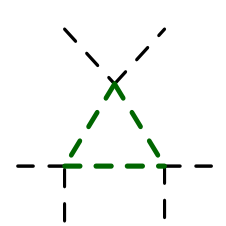

(vii)

Figure 3. Tree- (i)-(iv) and one-loop-level (v)-(viii) diagrams built of Lorentz invariant renormalizable interactions of the UV theory that lead to effective operators of $\phi^{6}$ class.

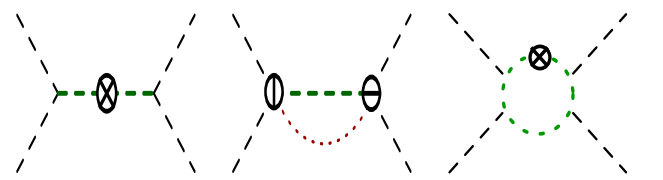

(i) (ii)<smiles>CC1(C)C[Te](C)(C)CC1(C)C</smiles>

(iv)

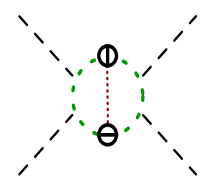

(v)

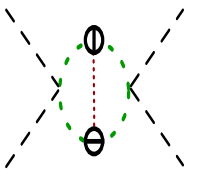

(vi)<smiles>CC1OC2(C)OC(C1C)C(C)C2C</smiles>

(vii)

Figure 4. Tree- (i)-(ii) and one-loop-level (iii)-(v) diagrams built of Lorentz invariant renormalizable interactions of the UV theory that lead to effective operators of $\phi^{4} \mathcal{D}^{2}$ class.

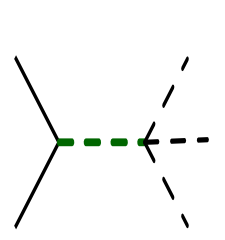

(i)

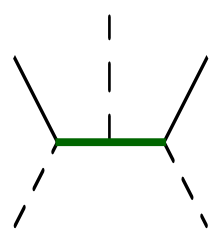

(ii)

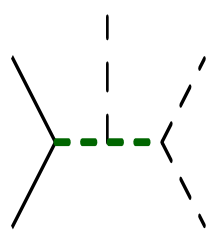

(iii)

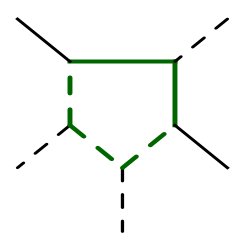

(iv)

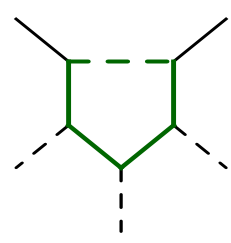

(v)

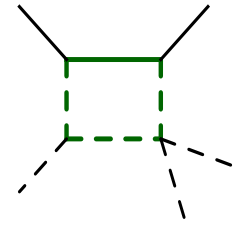

(vi)

Figure 5. Tree- (i)-(iii) and one-loop-level (iv)-(vii) diagrams built of Lorentz invariant renormalizable interactions of the UV theory that lead to effective operators of $\psi^{2} \phi^{3}$ class.

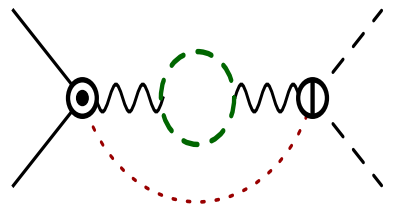

(i)

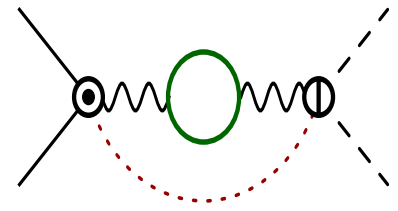

(ii)

Figure 6. One-loop diagrams built of Lorentz invariant renormalizable interactions of the UV theory that lead to effective operators of $\psi^{2} \phi^{2} \mathcal{D}$ class.

\section{(i) Cataloguing an exhaustive list of heavy field representations.}

- Based on figure 1, we have listed all possible combinations of light and heavy fields that lead to a particular vertex. In the rest of this paper, these have been referred to as "fundamental vertices".

- For each vertex and its sub-cases, based on the fixed light fields, we have identified all possible representations of the heavy fields. The necessary group-theoretic multiplications have been described in appendix B. We have presented the results for vertices composed of scalars, fermions, gauge bosons and their combinations in tables 2-4. 


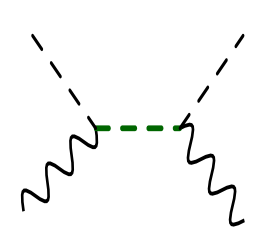

(i)<smiles>[Z6]C1C(C)C(C)C1N</smiles>

(iii)

(iv)<smiles>CC1CC(C)C(N)C(C)C1N</smiles>

(v)

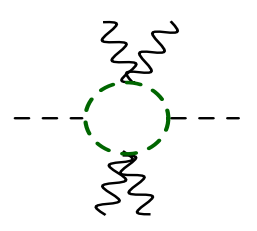

(vi)<smiles>CCC1CC(C)CC(C)CC(C)C1</smiles>

(vii)<smiles>CCC1(C)CC(C)C(C)CC(C)C(C)C1</smiles>

(viii)<smiles>[Z]C1CC(CCN)C(C)C(C)CC(CC)C1C</smiles>

(ix)<smiles>CCC1CCC(C)(C)CCC1CCN</smiles>

(x)<smiles>CC(C)(C)C1CCC(C)(C)C(C)(C)CC1NCN</smiles>

(xi)

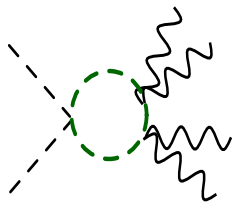

(xii)

Figure 7. Tree- (i)-(ii) and one-loop-level (iii)-(viii) diagrams built of Lorentz invariant renormalizable interactions of the UV theory that lead to effective operators of $\phi^{2} X^{2}$ class.

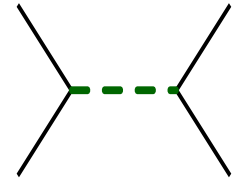

(i)

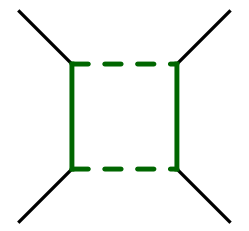

(ii)

Figure 8. (i) Tree- and (ii) one-loop-level diagrams built of Lorentz invariant renormalizable interactions of the UV theory that lead to effective operators of $\psi^{4}$ class.

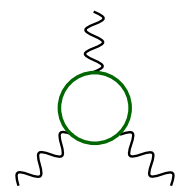

(i)

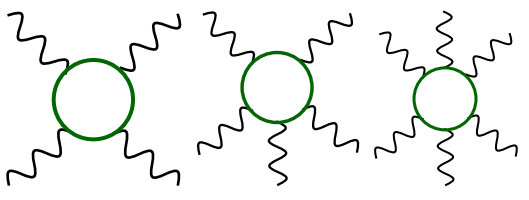

(ii)

(iii)

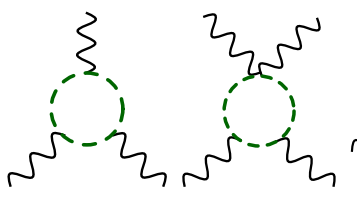

(vi)

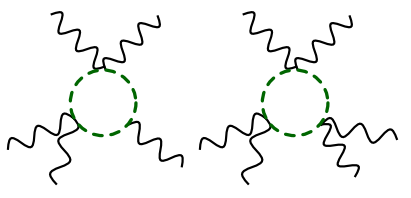

(vii) (viii)

Figure 9. One-loop diagrams built of Lorentz invariant renormalizable interactions of the UV theory that lead to effective operators of $X^{3}$ class.

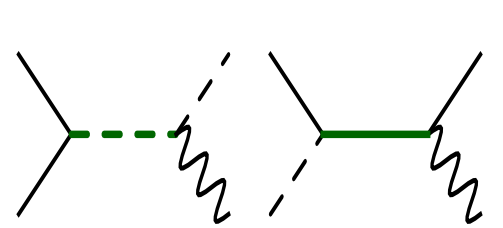

(i)

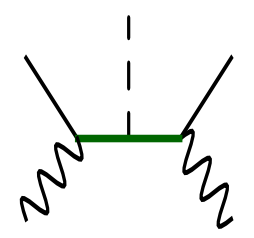

(iii)

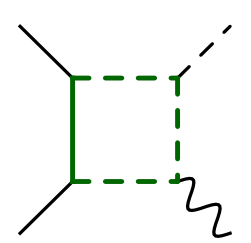

(iv)

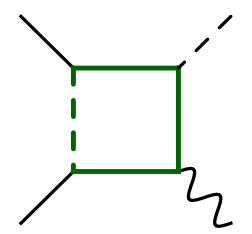

(v)

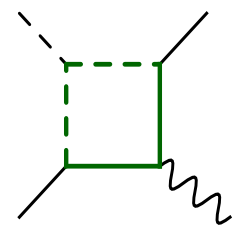

(vi)

Figure 10. Tree- (i)-(iv) and one-loop-level (v)-(ix) diagrams built of Lorentz invariant renormalizable interactions of the UV theory that lead to effective operators of $\psi^{2} \phi X$ class. 


\begin{tabular}{|c|c|c|c|}
\hline Vertex & S. No. & Light fields & Heavy field(s) \\
\hline${ }_{\phi_{2}}^{\phi_{1}},{ }^{\prime}$, & V1-(i) & $\begin{array}{c}\phi_{1}=\phi_{2}=H_{\left(1,2, \frac{1}{2}\right)} \text { or } H_{\left(1,2,-\frac{1}{2}\right)}^{\dagger} \\
\phi_{1}=H, \quad \phi_{2}=H^{\dagger}\end{array}$ & $\begin{array}{l}\Phi_{3} \in\{(1,3, \pm 1),(1,1, \pm 1)\} \\
\Phi_{3} \in\{(1,3,0),(1,1,0)\}\end{array}$ \\
\hline$\Phi_{2}$ & $\mathrm{~V} 2$ & $\phi_{1}=H$ or $H^{\dagger}$ & $\begin{array}{c}\Phi_{2} \in\left(R_{C_{2}}, R_{L_{2}}, Y_{2}\right), \Phi_{3} \in\left(R_{C_{3}}, R_{L_{3}}, Y_{3}\right) \\
\text { with } R_{C_{2}} \otimes R_{C_{3}} \equiv 1, R_{L_{2}} \otimes R_{L_{3}} \equiv 2 \\
\text { and } Y_{2}+Y_{3}= \pm \frac{1}{2}\end{array}$ \\
\hline${ }_{\phi_{2}, \prime^{\prime}}^{\phi_{1}}{ }^{\prime} x^{\prime},{ }^{\Phi_{4}}$ & V3-(i) & $\begin{array}{c}\phi_{1}=\phi_{2}=\phi_{3}=H \text { or } H^{\dagger} \\
\phi_{1}=\phi_{2}=H, \quad \phi_{3}=H^{\dagger}\end{array}$ & $\begin{array}{c}\Phi_{4} \in\left\{\left(1,4, \pm \frac{3}{2}\right),\left(1,2, \pm \frac{3}{2}\right)\right\} \\
\Phi_{4} \in\left\{\left(1,4, \pm \frac{1}{2}\right),\left(1,2, \pm \frac{1}{2}\right)\right\}\end{array}$ \\
\hline 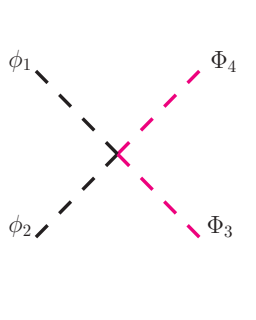 & V4-(i) & $\begin{array}{c}\phi_{1}=H, \quad \phi_{2}=H^{\dagger} \\
-------------\end{array}$ & $\begin{array}{c}\Phi_{3} \in\left(\left\{1, R_{C}\right\},\left\{1, R_{L}\right\},\{0, Y\}\right), \Phi_{4}=\Phi_{3}^{\dagger} \\
\Phi_{3} \in\left(R_{C_{3}}, R_{L_{3}}, Y_{3}\right), \Phi_{4} \in\left(R_{C_{4}}, R_{L_{4}}, Y_{4}\right) \\
\text { with } R_{C_{3}} \otimes R_{C_{4}} \equiv 1, R_{L_{3}} \otimes R_{L_{4}} \equiv 1 \text { or } 3 \\
\text { and } Y_{3}+Y_{4}= \pm 1 .\end{array}$ \\
\hline
\end{tabular}

Table 2. Allowed heavy field representations when the light degrees of freedom are the Standard Model ones. Here, $\phi_{i}$ denote the SM Higgs and $\Phi_{i}$ the various heavy scalars. $R_{C}$ and $R_{L}$ denote representations under $\mathrm{SU}(3)_{C}$ and $\mathrm{SU}(2)_{L}$ gauge groups and $Y$ refers to the $\mathrm{U}(1)_{Y}$ hypercharge of the field. Their appearance describes the cases where the vertex is not constituted by a unique heavy field representation but can involve a plethora of them.

\section{(ii) Unfolding effective operators in terms of UV diagrams.}

Having obtained an exhaustive list of heavy field representations based on the renormalizable vertices, we can focus our attention on ascertaining the correspondence between these heavy fields and the dimension-6 $C P$ as well as $B$-, $L$ - conserving SMEFT operators. We have accomplished this through the following steps.

- We have unfolded the operators, on a case-by-case basis, into tree- and one-looplevel diagrams describing processes of the UV theory and revealing various vertices of tables $2-4$ as the constituents of those diagrams for different cases.

- However, we must emphasize that instead of conducting the unfolding exhaustively by drawing the whole myriad of diagrams shown in figures 3-6 relevant for each operator and then tracing the possible heavy fields within those diagrams, we have 


\begin{tabular}{|c|c|c|c|}
\hline Vertex & S. No. & Light fields & Heavy field(s) \\
\hline \multirow{15}{*}{${ }^{\psi_{1}}$} & V5-(i) & $\psi_{1}=\psi_{2}=e_{(1,1,-1)}$ & $\Phi \in(1,1,2)$ \\
\hline & V5-(ii) & $\psi_{1}=\psi_{2}=l_{\left(1,2,-\frac{1}{2}\right)}$ & $\Phi \in\{(1,1,1),(1,3,1)\}$ \\
\hline & V5-(iii) & $\psi_{1}=\psi_{2}=d_{\left(3,1,-\frac{1}{3}\right)}$ & $\Phi \in\left\{\left(3,1, \frac{2}{3}\right),\left(\overline{6}, 1, \frac{2}{3}\right)\right\}$ \\
\hline & V5-(iv) & $\psi_{1}=\psi_{2}=u_{\left(3,1, \frac{2}{3}\right)}$ & $\Phi \in\left\{\left(3,1,-\frac{4}{3}\right),\left(\overline{6}, 1,-\frac{4}{3}\right)\right\}$ \\
\hline & V5-(v) & $\psi_{1}=\psi_{2}=q_{\left(3,2, \frac{1}{6}\right)}$ & $\Phi \in\left\{\left(3,1,-\frac{1}{3}\right),\left(3,3,-\frac{1}{3}\right),\left(\overline{6}, 1,-\frac{1}{3}\right),\left(\overline{6}, 3,-\frac{1}{3}\right)\right\}$ \\
\hline & V5-(vi) & $\left(\psi_{1}, \psi_{2}\right)=(\bar{l}, e)$ & $\Phi \in\left(1,2, \frac{1}{2}\right)$ \\
\hline & V5-(vii) & $\left(\psi_{1}, \psi_{2}\right)=(\bar{q}, d)$ & $\Phi \in\left\{\left(1,2, \frac{1}{2}\right),\left(8,2, \frac{1}{2}\right)\right.$ \\
\hline & V5-(viii) & $\left(\psi_{1}, \psi_{2}\right)=(\bar{u}, q)$ & $\Phi \in\left\{\left(1,2, \frac{1}{2}\right),\left(8,2, \frac{1}{2}\right)\right\}$ \\
\hline & V5-(ix) & $\left(\psi_{1}, \psi_{2}\right)=(q, l)$ & $\Phi \in\left\{\left(\overline{3}, 1, \frac{1}{3}\right),\left(\overline{3}, 3, \frac{1}{3}\right)\right.$ \\
\hline & V5-(x) & $\left(\psi_{1}, \psi_{2}\right)=(u, d)$ & $\Phi \in\left\{\left(3,1,-\frac{1}{3}\right),\left(\overline{6}, 1,-\frac{1}{3}\right)\right\}$ \\
\hline & V5-(xi) & $\left(\psi_{1}, \psi_{2}\right)=(u, e)$ & $\Phi \in\left(\overline{3}, 1, \frac{1}{3}\right)$ \\
\hline & V5-(xii) & $\left(\psi_{1}, \psi_{2}\right)=(d, e)$ & $\Phi \in\left(\overline{3}, 1, \frac{4}{3}\right)$ \\
\hline & V5-(xiii) & $\left(\psi_{1}, \psi_{2}\right)=(\bar{q}, e)$ & $\Phi \in\left(3,2, \frac{7}{6}\right)$ \\
\hline & V5-(xiv) & $\left(\psi_{1}, \psi_{2}\right)=(\bar{l}, u)$ & $\Phi \in\left(\overline{3}, 2,-\frac{7}{6}\right)$ \\
\hline & V5-(xv) & $\left(\psi_{1}, \psi_{2}\right)=(\bar{l}, d)$ & $\Phi \in\left(\overline{3}, 2,-\frac{1}{6}\right)$ \\
\hline \multirow{10}{*}{$\begin{array}{c}\left.{ }^{\psi}\right\rangle \\
! \\
,\end{array}$} & V6-(i) & $\phi=H_{\left(1,2, \frac{1}{2}\right)}, \quad \psi=l$ & $\Psi \in\{(1,1,0),(1,3,0)\}$ \\
\hline & V6-(ii) & $\phi=H, \quad \psi=e$ & $\Psi \in\left(1,2, \frac{1}{2}\right)$ \\
\hline & V6-(iii) & $\phi=H, \quad \psi=q$ & $\Psi \in\left\{\left(\overline{3}, 1,-\frac{2}{3}\right),\left(\overline{3}, 3,-\frac{2}{3}\right)\right\}$ \\
\hline & V6-(iv) & $\phi=H, \quad \psi=u$ & $\Psi \in\left(\overline{3}, 2,-\frac{7}{6}\right)$ \\
\hline & V6-(v) & $\phi=H, \quad \psi=d$ & $\Psi \in\left(\overline{3}, 2,-\frac{1}{6}\right)$ \\
\hline & V6-(vi) & $\phi=H^{\dagger}, \quad \psi=l$ & $\Psi \in\{(1,1,1),(1,3,1)\}$ \\
\hline & V6-(vii) & $\phi=H^{\dagger}, \quad \psi=e$ & $\Psi \in\left(1,2, \frac{3}{2}\right)$ \\
\hline & V6-(viii) & $\phi=H^{\dagger}, \quad \psi=q$ & $\Psi \in\left\{\left(\overline{3}, 1, \frac{1}{3}\right),\left(\overline{3}, 3, \frac{1}{3}\right)\right\}$ \\
\hline & V6-(ix) & $\phi=H^{\dagger}, \quad \psi=u$ & $\Psi \in\left(\overline{3}, 2,-\frac{1}{6}\right)$ \\
\hline & V6-(x) & $\phi=H^{\dagger}, \quad \psi=d$ & $\Psi \in\left(\overline{3}, 2, \frac{5}{6}\right)$ \\
\hline
\end{tabular}

Table 3. Table 2 continued. Here, $\phi_{i}$ and $\psi_{i}$ denote the SM Higgs and the SM fermions respectively, whereas $\Phi_{i}$ and $\Psi_{i}$ denote the various heavy scalars and heavy fermions. 


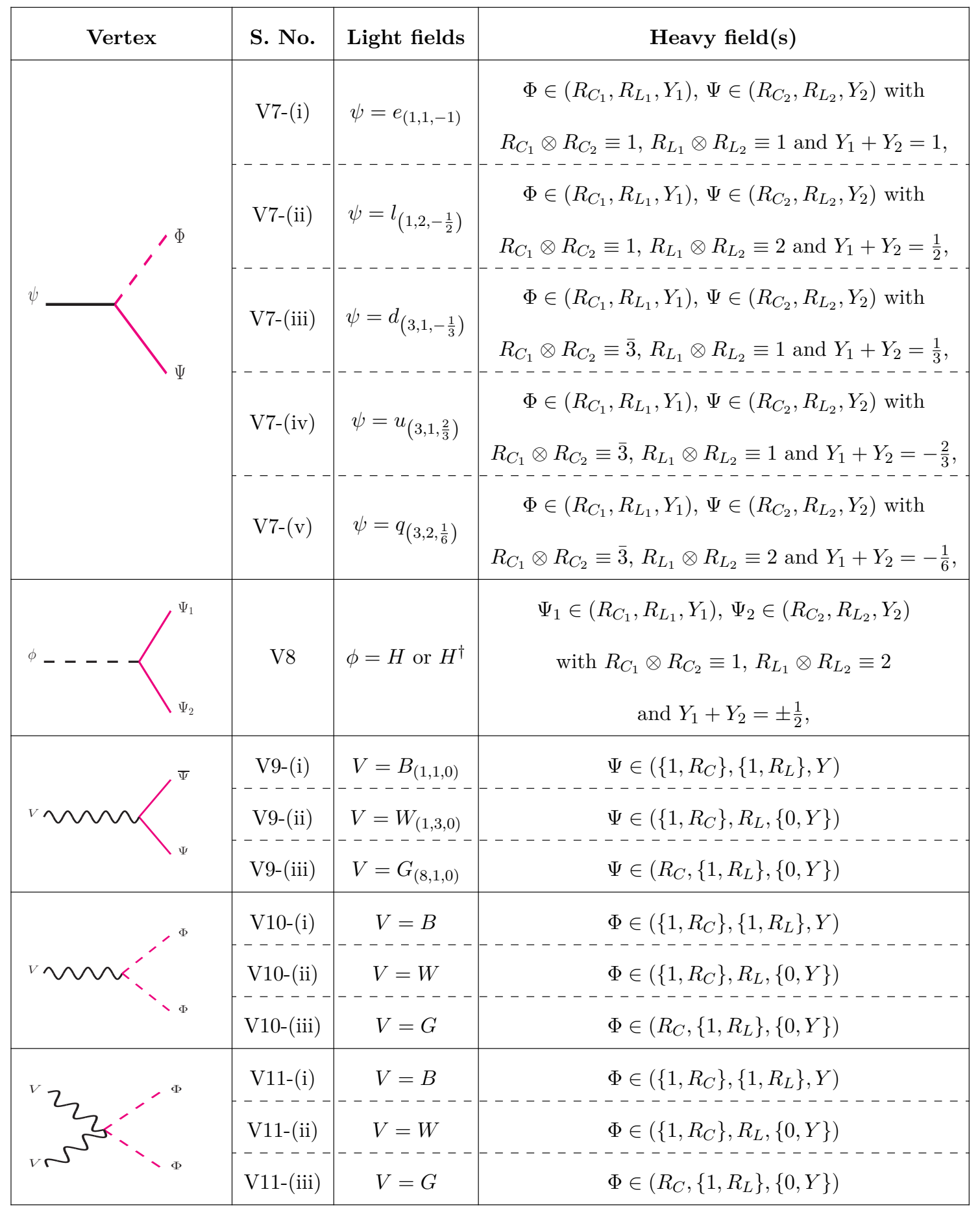

Table 4. Table 3 continued. Here, $\phi_{i}, \psi_{i}$ and $V$ denote the SM Higgs, the SM fermions and the SM gauge bosons respectively, whereas $\Phi_{i}$ and $\Psi_{i}$ denote the various heavy scalars and heavy fermions. $R_{C}$ and $R_{L}$ denote $\mathrm{SU}(3)_{C}$ and $\mathrm{SU}(2)_{L}$ quantum numbers respectively and $Y$ refers to the $\mathrm{U}(1)_{Y}$ hypercharge of the field. 
followed a well defined and economical approach. We have searched for the most minimal way in which it can be shown that a particular operator receives non-zero contributions from a single heavy field.

- Noting the fact that a particular heavy field can very well contribute to the same operator through multiple channels, we have included only one relevant diagram ${ }^{4}$ exhibiting the connection between the two for every combination of heavy field and effective operator.

- For individual operators, we have included diagrams built of only one kind of fundamental vertex and containing a single type of heavy field propagator among the internal lines.

- While we have found this prescription to work for operator classes which are composed of one type of fields, e.g., the $\phi^{6}, X^{3}$ classes and certain operators of the $\psi^{4}$ class, we have been forced to relax the strict minimality criteria once we start examining operator classes of non-trivial constitution. The shift from our notion of minimality is necessary for the following scenarios:

- In situations where the number of heavy fields is still one but due to the appearance of different SM fields as the external legs and the variety of ways in which these legs can be permuted, the number of fundamental vertices involved in the diagram is more than one. This has also been observed to lead to light-heavy mixing in the loop.

- Situations where in a particular diagram a single vertex demands multiple heavy field representations simultaneously. This mostly corresponds to those entries of tables 2-4 that lead to $\Phi_{i}, \Psi_{i} \in\left(R_{C_{i}}, R_{L_{i}}, Y_{i}\right)$ with strict conditions imposed on these quantum numbers depending on the vertex.

- More dramatic departures where not only multiple heavy fields but sometimes multi-loop diagrams also become necessary. Such cases have been addressed in section 4 .

- In passing, it must be mentioned that we have excluded the cases involving heavy gauge bosons. This is because our focus is on minimal extensions of the SM and we are not including scenarios that involve the breaking of some higher symmetry.

- We must emphasize that the results of tables $2-4$ are necessary in order to understand the results collected in tables $5-22$.

- Each of these tables contains a list of heavy fields that can be back-traced from a particular operator.

- Next to each operator we have attached the diagram it originates from. The diagrams can denote pure heavy propagators at tree- or one-loop-level, as well as light-heavy mixing in the loops

\footnotetext{
${ }^{4}$ Here, relevant refers to diagrams that yield non-zero contributions. Also, it must be noted that since we have not discussed the relative strengths of the coupling constants that may appear at particular vertices, all diagrams at a certain order have been treated on an equal footing.
} 
- Corresponding to each heavy field appearing from a diagram we have also listed the vertex or the group of vertices constituting the diagram that involves the particular heavy field.

- For ease of readability we have established hyperlinks to the entries of tables 2-4, so that one can suitably verify the appearance of the heavy field.

\section{$3.1 \phi^{6}$}

Being consistent with our idea of minimality, we have tried to identify heavy fields that can lead to $\mathcal{Q}_{H}=\left(H^{\dagger} H\right)^{3}$ through tree- and one-loop-level diagrams built of the smallest number of fundamental vertices listed in table 2 . We have collected our results in table 5 and the following are salient points related to the results:

- Our notion of minimality restricts us to the following diagrams:

1. Figure $3 \mathrm{i}$ - a tree-level diagram containing only quartic scalar vertices joined by a heavy propagator.

2. Figure 3vi - one-loop-level diagram containing only trilinear scalar vertices. This presents two distinct sub-cases involving

(a) light-heavy mixing in the loop between the SM scalar and the heavy scalar.

(b) mixing between two distinct heavy fields in the loop. This corresponds to the case where the UV theory has a degenerate spectrum. We have mostly avoided such cases in the remainder of this paper.

3. Figure 3vii - one-loop-level diagram containing only quartic scalar vertices. This contains both heavy-heavy mixing as well as a single heavy field in the loop.

- A simple inspection reveals that the heavy field representations obtained from figure 3vi do in fact appear at tree-level through figure 3ii but this has not been included here as it contains both trilinear as well as quartic scalar vertices, therefore not being a minimal option.

- Specific heavy field representations are fixed using the contents of table 2 as well as by noting the permutations of the external legs of the effective operator in the unfolded diagrams. For instance, if we consider the tree-level diagram in table 5, then different heavy fields emerge based on whether we have $H^{3}$ or $H^{2} H^{\dagger}$ on one of the vertices. To highlight these differences we have separated the multiple heavy fields embedded in the same diagram through a dashed line in the tables and we have also referenced the specific vertices involved next to them.

- It must be noted that in each diagram in tables 5-22, the black and pink lines represent light and heavy fields respectively.

\section{$3.2 \phi^{4} \mathcal{D}^{2}$}

This class contains two operators $\mathcal{Q}_{H \square}$ and $\mathcal{Q}_{H \mathcal{D}}$. In both cases, the external states contain 4 scalars and 2 derivatives. The difference is that in the first case the derivatives are Lorentz contracted together to form the $\mathcal{D}^{2}$ operator, whereas in the second case the derivatives 


\begin{tabular}{|c|c|c|c|c|c|}
\hline \multicolumn{6}{|c|}{$\mathcal{Q}_{H}:\left(H^{\dagger} H\right)^{3}$} \\
\hline Heavy fields & Diagram & Vertices & Heavy fields & Diagram & Vertices \\
\hline $\begin{array}{l}(1,3,1),(1,1,1) \\
(1,3,0),(1,1,0)\end{array}$ & & V1-(i) & $\begin{array}{r}\left(1,4, \frac{3}{2}\right),\left(1,2, \frac{3}{2}\right) \\
\left(1,4, \frac{1}{2}\right),\left(1,2, \frac{1}{2}\right)\end{array}$ & $\begin{array}{l}\phi_{1} \\
1 \\
-2 \\
1 \\
d_{3}\end{array}$ & $\begin{array}{l}\text { V3-(i) } \\
\text { V3-(ii) }\end{array}$ \\
\hline $\begin{array}{c}\left(R_{C_{2}}, R_{L_{2}}, Y_{2}\right) \oplus \\
\left(R_{C_{3}}, R_{L_{3}}, Y_{3}\right)\end{array}$ & & V2 & $\begin{array}{c}\left(\left\{1, R_{C}\right\},\left\{1, R_{L}\right\},\{0, Y\}\right) \\
\left(R_{C_{3}}, R_{L_{3}}, Y_{3}\right) \oplus \\
\left(R_{C_{4}}, R_{L_{4}}, Y_{4}\right)\end{array}$ & 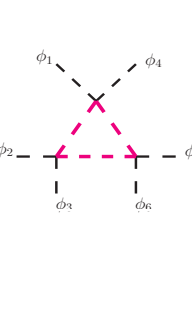 & V4-(i) \\
\hline
\end{tabular}

Table 5. Heavy field representations that are obtained by unfolding the $\phi^{6}$ operator into non-trivial tree- and (or) one-loop-level diagrams and the corresponding vertices.

act on separate fields. Due to this, we get an extra diagram for $\mathcal{Q}_{H \mathcal{D}}$ with heavy-heavy mixing of fermions in the loop. Tree level diagrams with a heavy scalar propagator and one-loop-level diagrams with heavy scalar as well as heavy-heavy mixing are common to both operators. Specifically, looking at figure 4, the following diagrams appear:

1. Tree-level diagrams: figures $4 \mathrm{i}$ and $4 \mathrm{ii}$ - Both diagrams contain trilinear scalar vertices and a scalar propagator but in the first case the derivatives are contracted together whereas in the second case they act on separate fields.

2. One-loop diagrams

(a) Figure 4iii — This diagram contains trilinear scalar vertices and a scalar loop with the derivatives contracted together.

(b) Figure 4iv - This diagram contains quartic scalar vertices and a scalar loop with the derivatives contracted together.

(c) Figure 4v - This diagram contains trilinear scalar vertices and a scalar loop with the derivatives acting on separate fields.

(d) Figure 4vi - This diagram contains quartic scalar vertices and a scalar loop with the derivatives acting on separate fields.

(e) Figure 4vii - This diagram contains Yukawa-like vertices and a fermion loop with the derivatives acting on separate fields. 


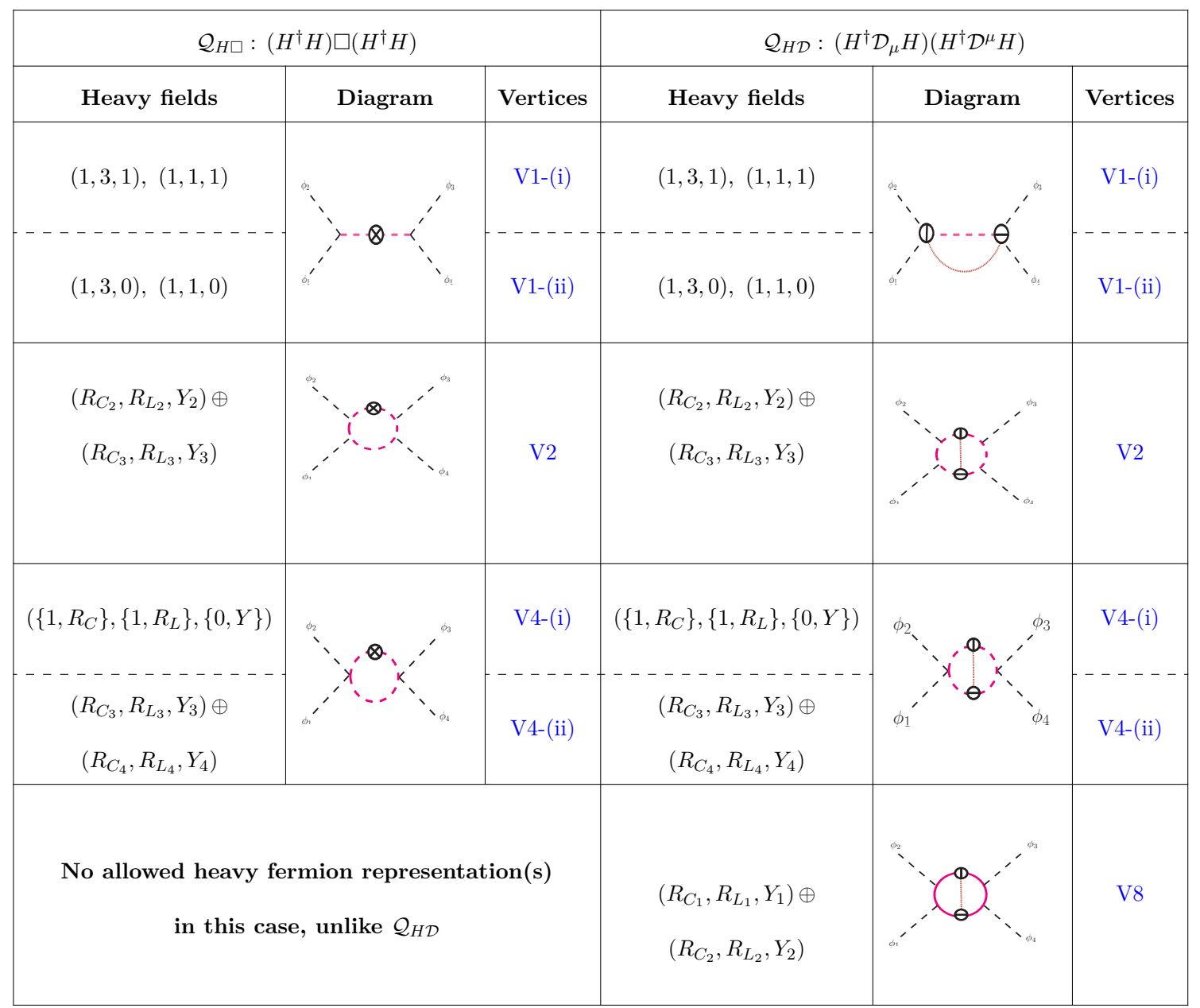

Table 6. Heavy field representations that are obtained by unfolding the $\phi^{4} \mathcal{D}^{2}$ operators into nontrivial tree- and (or) one-loop-level diagrams and the corresponding vertices. The entries for the operators $\mathcal{Q}_{H \square}$ and $\mathcal{Q}_{H \mathcal{D}}$ have been presented side by side to allow for an easy comparison between the two.

Figures $4 \mathrm{i}$, 4iii and $4 \mathrm{iv}$ contribute to $\mathcal{Q}_{H \square}$, while figures $4 \mathrm{ii}, 4 \mathrm{v}, 4 \mathrm{vi}$ and 4 vii contribute to $\mathcal{Q}_{H \mathcal{D}}$. Representations of the heavy fields are once again fixed using the contents of tables 2 and 4 . The detailed results have been collected in table 6 .

\section{$3.3 \psi^{2} \phi^{3}$}

This class contains 3 operators $-\mathcal{Q}_{d H}, \mathcal{Q}_{u H}$ and $\mathcal{Q}_{e H}$. Following the scheme of unfolding operators into tree and (or) loop-level diagrams constituted of invariant renormalizable, the emergence of heavy fields is minimally described by the following diagrams:

1. Figure 5i - It appears in all three operators and in all 3 cases furnishes a heavy scalar with identical quantum numbers as the SM Higgs, or in other words, the heavy scalar corresponds to the two-Higgs-doublet Model [58, 59]. The vertices involved are the Yukawa vertex and the quartic scalar vertex.

2. Figure $5 \mathrm{v}-$ This loop diagram appears in 3 different variations: 


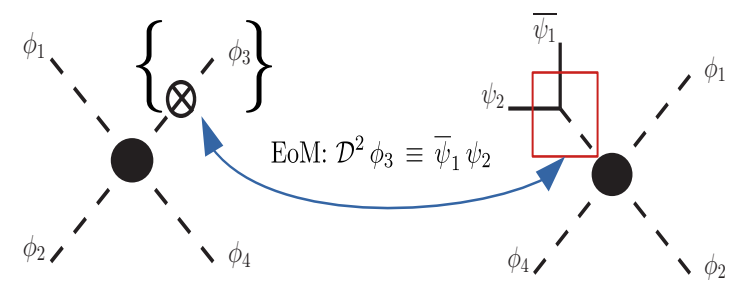

Figure 11. Implementing the equation of motion of the scalar field to relate $\left(H^{\dagger} H\right)\left(H^{\dagger} \mathcal{D}^{2} H\right)$ to operators of the $\psi^{2} \phi^{3}$ class.

(a) With a heavy scalar coupling with the SM fermions,

(b) With a single heavy fermion propagator in the loop coupling with the SM fermions as well as the SM scalar, and

(c) With 2 heavy fermion propagators in the loop, both having the same representations.

Specific results have been described in table 7 .

Taking equations of motion into account: the schematic effective operators for $\phi^{4} \mathcal{D}^{2}$ shown in figure 2vi can be interpreted in different ways. We have already discussed the cases where the individual derivatives, as well as the $\mathcal{D}^{2}$ operator, act on the heavy field. If we focus on the case where $\mathcal{D}^{2}$ acts on an external SM scalar, i.e., the operator $\left(H^{\dagger} H\right)\left(H^{\dagger} \mathcal{D}^{2} H\right)$, then based on the equation of motion [3, 60]:

$$
\mathcal{D}^{2} H \supset y_{e}^{p r} \bar{l}_{p} e_{r}+y_{d}^{p r} \bar{q}_{p} d_{r}+y_{u}^{p r} \bar{u}_{p} q_{r},
$$

we can establish a connection between this operator and operators of the $\psi^{2} \phi^{3}$ class. Figure 11 shows this connection schematically.

Due to this EOM, the heavy fields emerging from the unfolding of $\left(H^{\dagger} H\right)\left(H^{\dagger} \mathcal{D}^{2} H\right)$ into tree and loop level diagrams can, in fact, be connected to $\mathcal{Q}_{e H}, \mathcal{Q}_{d H}$ and $\mathcal{Q}_{u H}$. There are a couple of noteworthy points:

- The heavy fields which have now been connected to the $\psi^{2} \phi^{3}$ class are different from the ones obtained when individual operators of this class were unfolded into diagrams using renormalizable vertices.

- Heavy field representations discussed previously in table 7 involve certain cases which correspond to only one of the three operators $\mathcal{Q}_{e H}, \mathcal{Q}_{d H}$ or $\mathcal{Q}_{u H}$ but heavy fields obtained from the unfolding of $\left(H^{\dagger} H\right)\left(H^{\dagger} \mathcal{D}^{2} H\right)$ relate simultaneously to all three $\psi^{2} \phi^{3}$ operators.

In table 8, we have listed the heavy fields appearing through the following diagrams:

1. Tree level diagram with a single heavy propagator between trilinear scalar vertices.

2. One-loop diagram comprised only of trilinear scalar vertices and involving light-heavy mixing in the loop. 


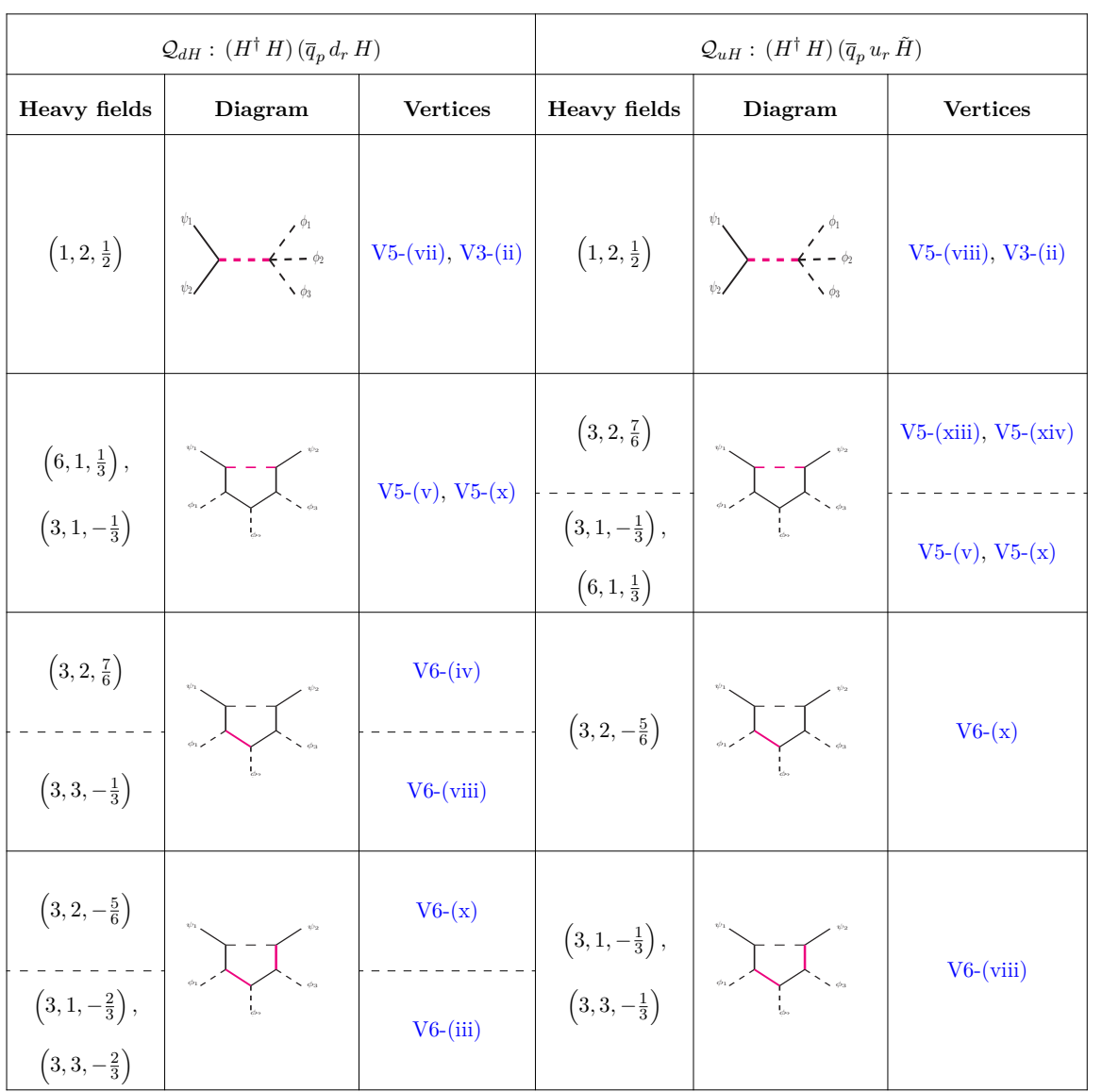

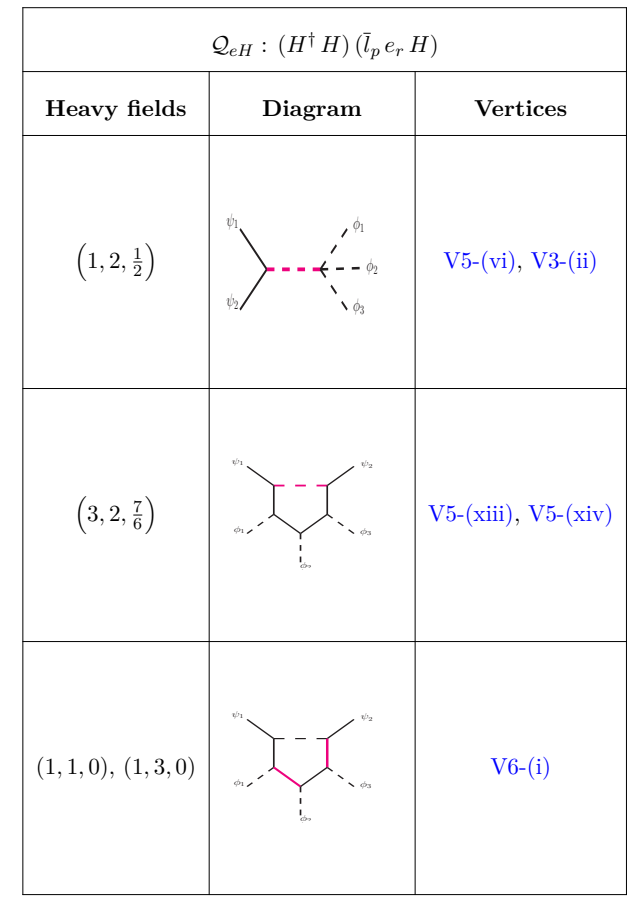

Table 7. Heavy field representations that are obtained by unfolding the $\psi^{2} \phi^{3}$ operators into nontrivial tree- and (or) one-loop-level diagrams and the corresponding vertices. 
3. One-loop diagram comprised only of Yukawa vertices and involving light-heavy mixing among fermions in the loop.

4. One-loop diagram comprised of quartic scalar vertices and a heavy loop.

\section{$3.4 \psi^{2} \phi^{2} \mathcal{D}$}

The presence of $\mathcal{D}_{\mu}$ and $\gamma^{\mu}$ within the effective operators of this class, see figure 2iv, indicates that the diagrams leading to these operators must involve vector bosons. Since we are not taking into account scenarios involving heavy gauge bosons, the heavy scalar or fermion appears in a loop and couples to the SM gauge bosons which in turn couple with the external states, see figures $6 \mathrm{i}$ and 6ii. Representations of the heavy field are fixed as follows:

- Choice of the external fermion in the effective operator determines the intermediate vector boson. $\mathrm{SU}(2)$ singlet fermions couple only with $B_{\mu}$, whereas $\mathrm{SU}(2)$ doublets couple with both $B_{\mu}$ and $W_{\mu}^{I}$. Since the $\mathrm{SM}$ scalar is $\mathrm{SU}(3)$ singlet, $G_{\mu}^{A}$ does not appear.

- Fixing the gauge boson ultimately sets the heavy field representations in accordance with the results of table 4 .

Results have been collected in table 9 .

\section{$3.5 \phi^{2} X^{2}$}

Given the external legs of the effective operator, see figure $2 \mathrm{v}$, and keeping in mind that there is no mixing in the kinetic sector of the renormalizable Lagrangian, heavy fields emerge only through one-loop-level diagrams, more specifically:

- Figure 7iv, which involves trilinear scalar vertices and vertices that couple scalars with gauge bosons. Heavy fields appear through light-heavy mixing within the loop, and the vector bosons coupling with the light field.

- Figure 7vii involves Yukawa like vertices connecting the SM scalar with two fermions and vertices emerging from fermion kinetic terms. Heavy fields once again emerge through light-heavy mixing but we encounter two distinct cases:

1. Vector bosons coupling with the light (SM) fermion.

2. Vector bosons coupling with the heavy fermion.

- Figure 7x contains quartic scalar interaction as well as coupling between scalars and gauge bosons. The heavy field appears in the loop (with no mixing).

In each case, the choice of the vector bosons, as well as the light SM fields in the loop, dictates the allowed representations of the heavy fields. We have collected our results in tables 10 and 11 . 


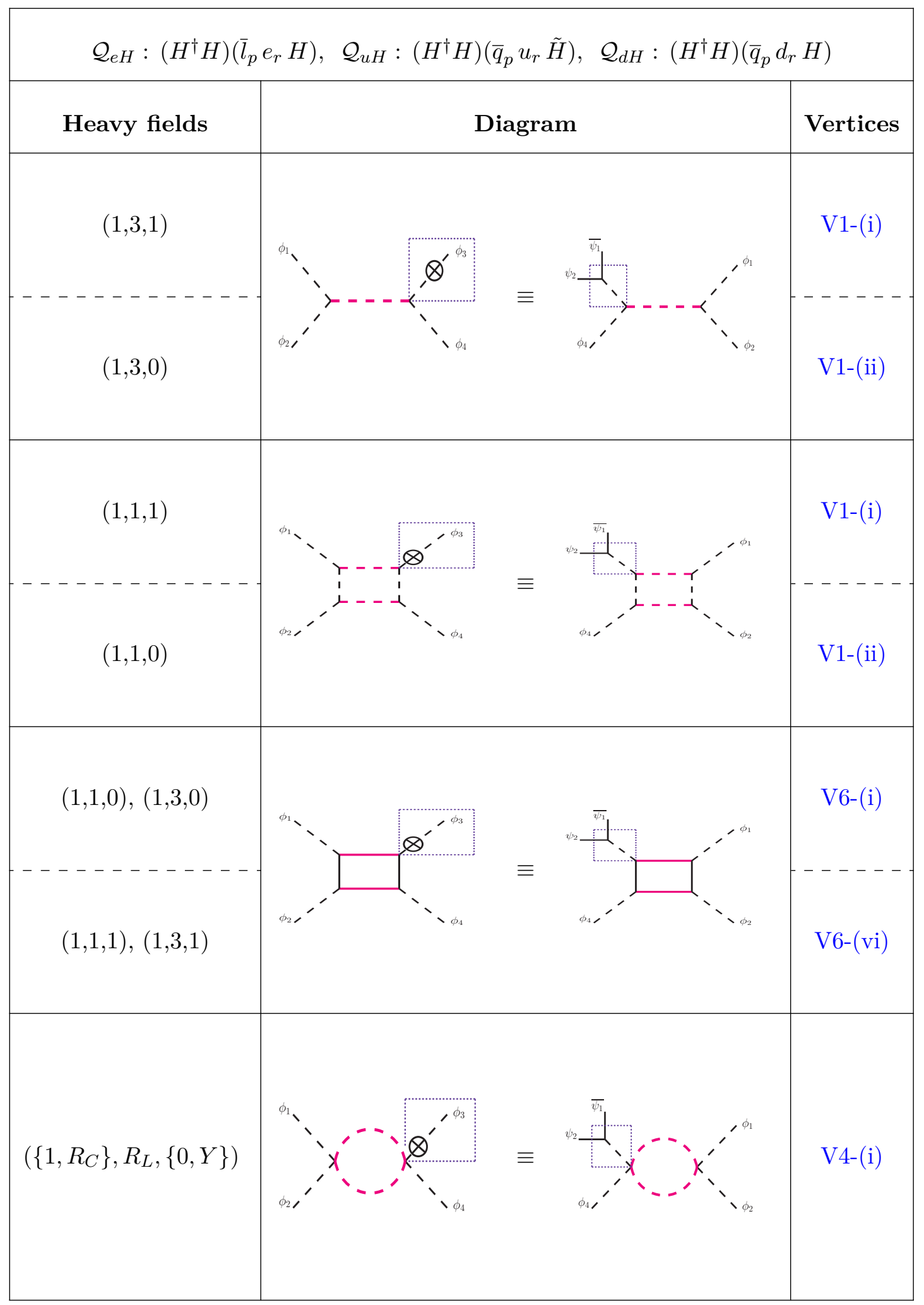

Table 8. Heavy field representations that indirectly provide non-zero contributions to the $\psi^{2} \phi^{3}$ operators when the equation of motion of the SM Higgs is implemented, in the process of unfolding $\phi^{4} \mathcal{D}^{2}$ class of operators. Also, listed are the vertices corresponding to the diagrams. 


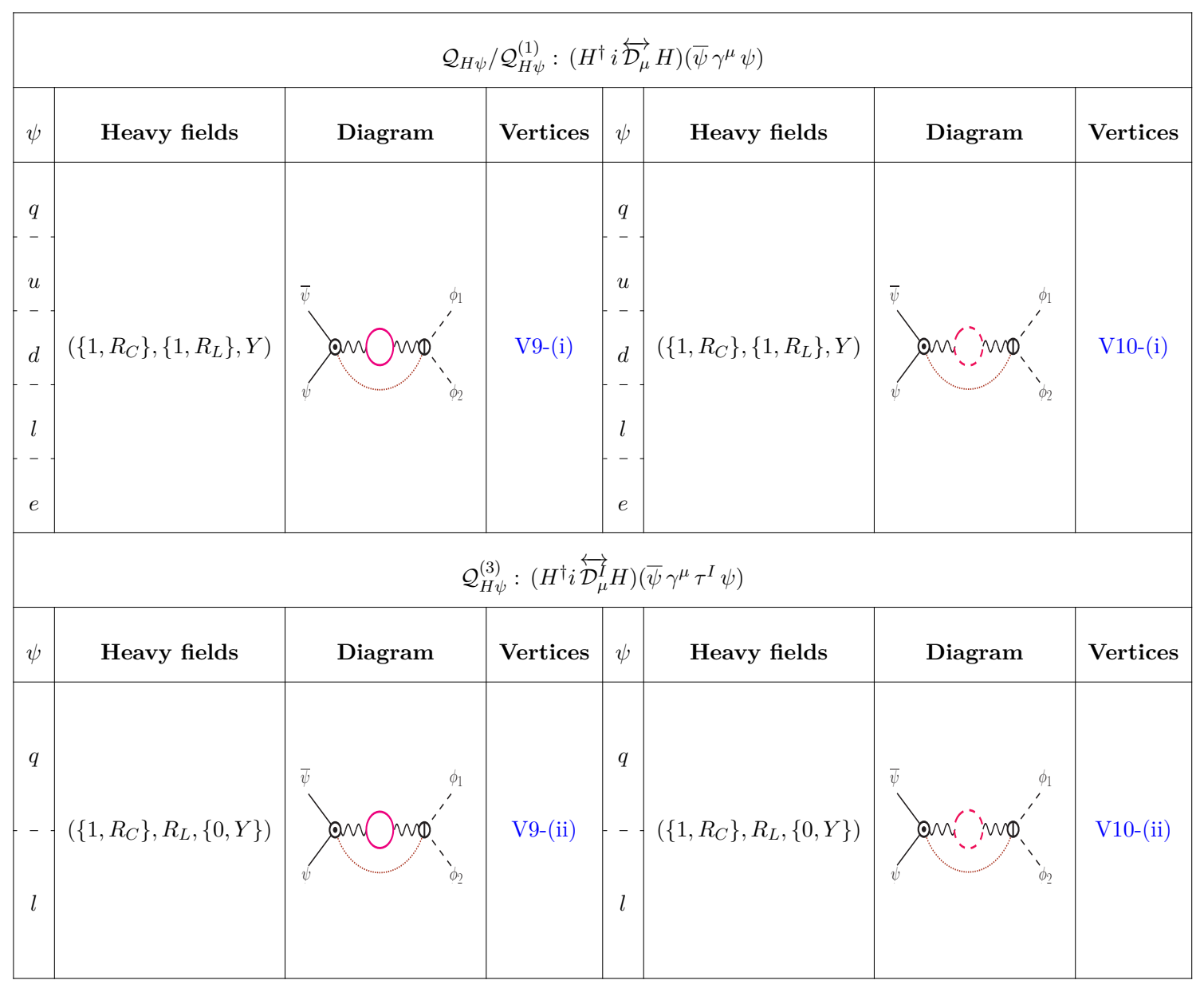

Table 9. Heavy field representations that are obtained by unfolding the $\psi^{2} \phi^{2} \mathcal{D}$ operators into non-trivial one-loop-level diagrams and the corresponding vertices. In each sub-table, operators with different fermions as external legs but leading to identical heavy field representations through similar diagrams have been grouped together. The headers schematically describe the covariant forms of the operators.

\section{$3.6 \psi^{4}$}

Restricting ourselves to only baryon and lepton number conserving operators, we can subdivide the $\psi^{4}$ operators into several sub-categories based on the chirality of the fields constituting the individual operators. The representations of the heavy fields from which the origin of these operators can be retraced also vary based on the kind of fermion bilinears that can be identified within each operator.

(i) $(\boldsymbol{L} \overline{\boldsymbol{L}})(\boldsymbol{L} \overline{\boldsymbol{L}}):$ this subclass contains operators constituted solely of the isospin doublet fermions $l, q$ and their conjugates, i.e., the operators $-\mathcal{Q}_{l l}, \mathcal{Q}_{l q}^{(1)}, \mathcal{Q}_{l q}^{(3)}, \mathcal{Q}_{q q}^{(1)}, \mathcal{Q}_{q q}^{(3)}$. In the Warsaw basis [3], these operators are expressed as the Lorentz contraction of two 4-vectors each of them being fermion bilinears of the form $\bar{\psi} \gamma_{\mu} \psi$. This also indicates that unfolding these operators must incorporate the SM vector bosons and heavy fields appear in the form of scalar or fermion loops coupling to these vector bosons. 


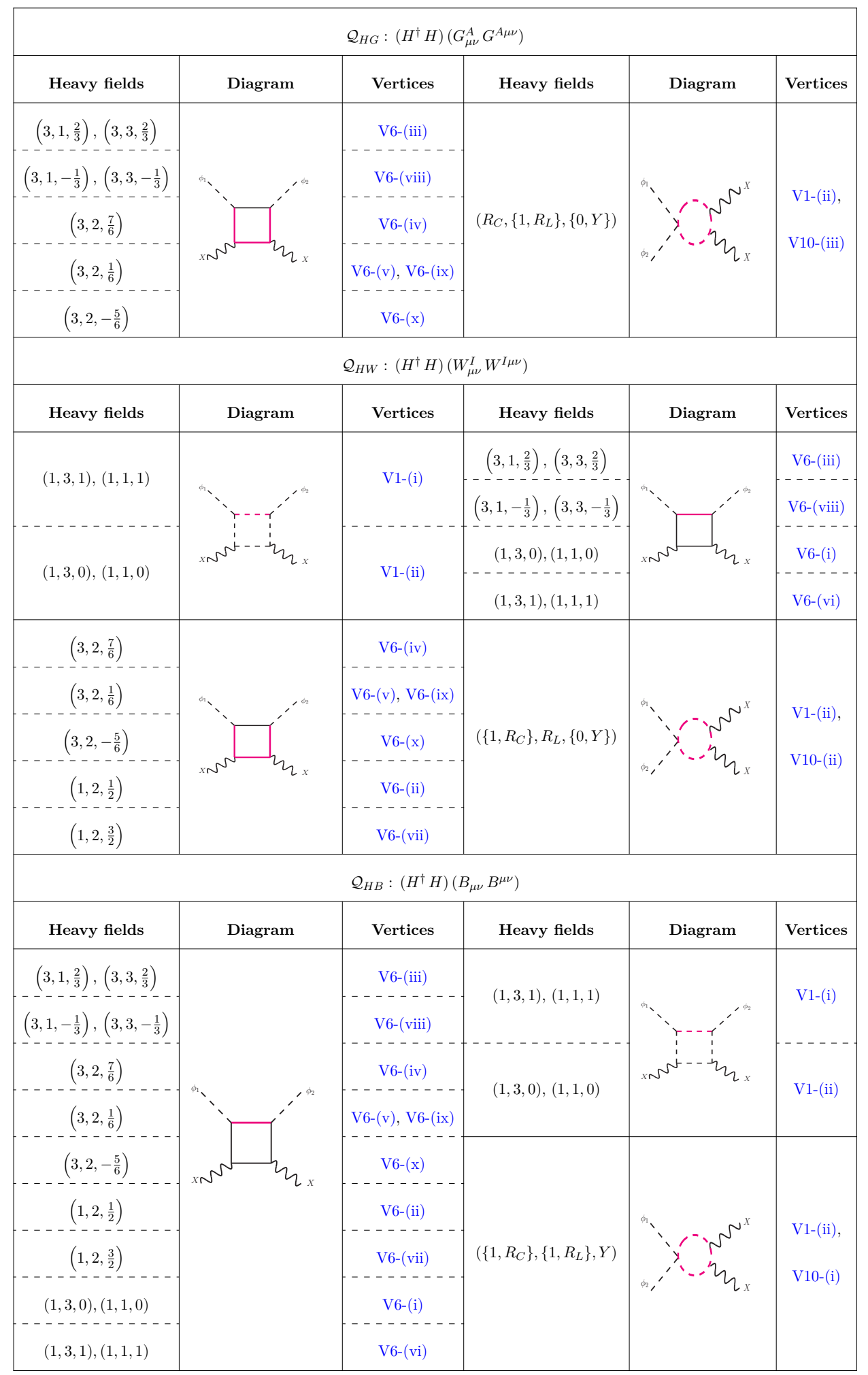

Table 10. Heavy field representations that are obtained by unfolding the $\phi^{2} X^{2}$ operators into non-trivial one-loop-level diagrams and the corresponding vertices. 


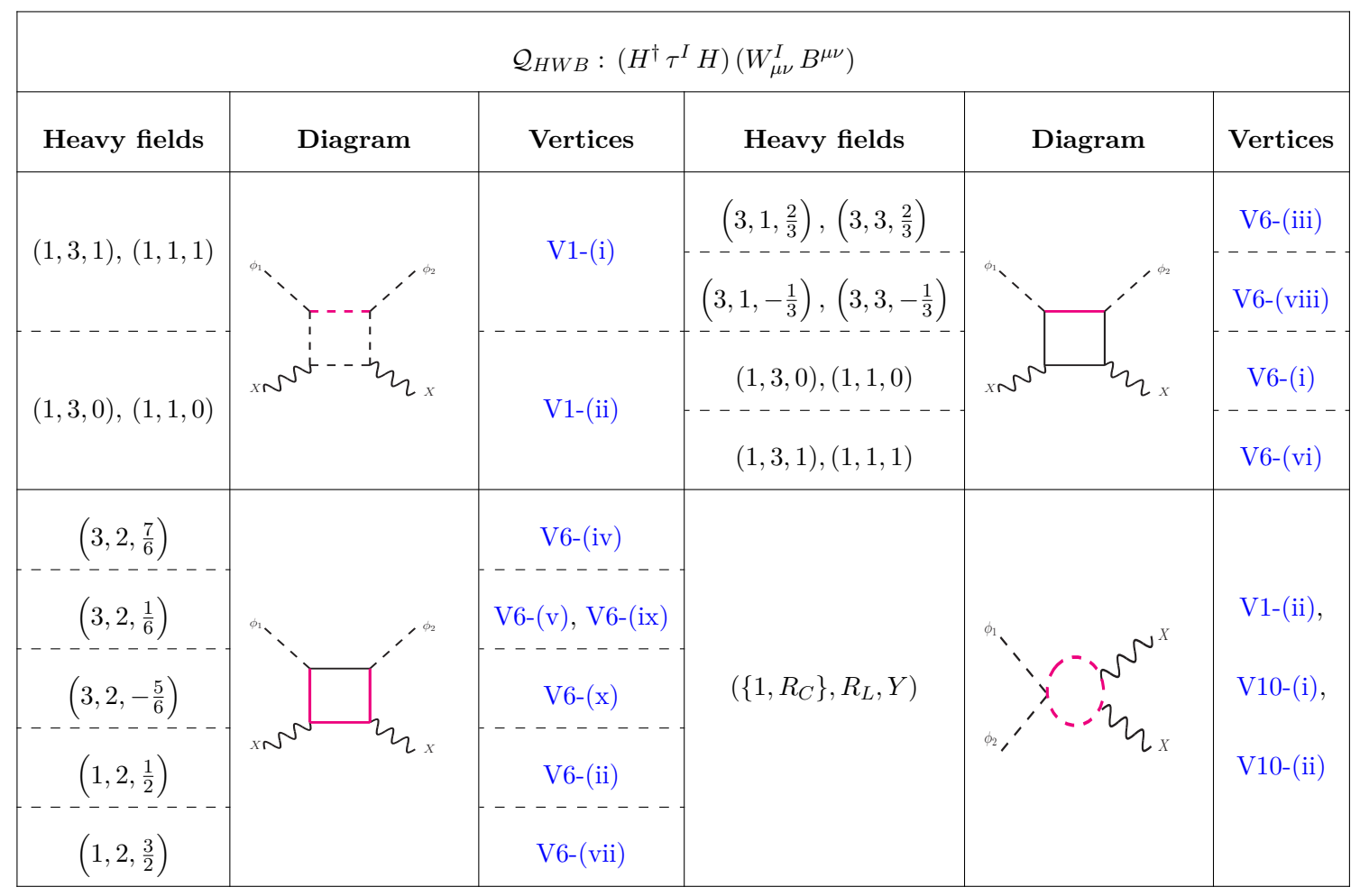

Table 11. Table 10 continued.

The choice of the vector boson is based on whether the fermion bilinears in the particular operator transform as singlets or as triplets under weak isospin $\mathrm{SU}(2)$. Therefore, $\mathcal{Q}_{l l}$, $\mathcal{Q}_{l q}^{(1)}$ and $\mathcal{Q}_{q q}^{(1)}$ involve $B_{\mu}$ whereas $\mathcal{Q}_{l q}^{(3)}$ and $\mathcal{Q}_{q q}^{(3)}$ (whose constituent fermion bilinears have the form $\bar{\psi} \gamma_{\mu} \tau^{I} \psi$ ) involve $W_{\mu}^{I}$ in their respective unfolded diagrams. Fixing the vector boson ultimately fixes the representations in accordance with the results of table 4 . The presence of an SM vector boson at each vertex also enforces the conservation of fermion flavour.

One can note the absence of an operator $\mathcal{Q}_{l l}^{(3)}$ of the form $\left(\bar{l}_{p} \gamma^{\mu} \tau^{I} l_{r}\right)\left(\bar{l}_{s} \gamma_{\mu} \tau^{I} l_{t}\right)$ by examining the following equations:

$$
\begin{aligned}
& \left(\bar{l}_{p} \gamma^{\mu} \tau^{I} l_{r}\right)\left(\bar{l}_{s} \gamma_{\mu} \tau^{I} l_{t}\right)=\left(\bar{l}_{p} \gamma^{\mu} l_{t}\right)\left(\bar{l}_{s} \gamma_{\mu} l_{r}\right)-\frac{1}{2}\left(\bar{l}_{p} \gamma^{\mu} l_{r}\right)\left(\bar{l}_{s} \gamma_{\mu} l_{t}\right), \\
& \left(\bar{l}_{p} \gamma^{\mu} \tau^{I} l_{r}\right)\left(\bar{q}_{s} \gamma_{\mu} \tau^{I} q_{t}\right)=\left(\bar{l}_{p} \gamma^{\mu} q_{t}\right)\left(\bar{q}_{s} \gamma_{\mu} l_{r}\right)-\frac{1}{2}\left(\bar{l}_{p} \gamma^{\mu} l_{r}\right)\left(\bar{q}_{s} \gamma_{\mu} q_{t}\right) .
\end{aligned}
$$

Here, we have used the Fierz relations for the SU(2) generators $\left(\tau^{I}\right)^{i}{ }_{j}\left(\tau^{I}\right)^{k}{ }_{l}=\delta^{i}{ }_{l} \delta^{k}{ }_{j}-$ $\frac{1}{2} \delta^{i}{ }_{j} \delta^{k}{ }_{l}$. In the first equation, both terms on the right are identical with just the flavour indices shuffled. Thus, the first equation only relates two quantities so the operator basis can only include one of these. On the other hand, the second equation connects three different quantities, hence the operator basis can only include two of these. This explains why there are two operators $\mathcal{Q}_{l q}^{(1),(3)}$ with the same external states but only one operator $\mathcal{Q}_{l l}$. And consequently, the absence of $W_{\mu}^{I}$ in the diagrams with $l$ and $\bar{l}$ as external lines on both vertices is explained. A similar argument explains the absence of an operator of 


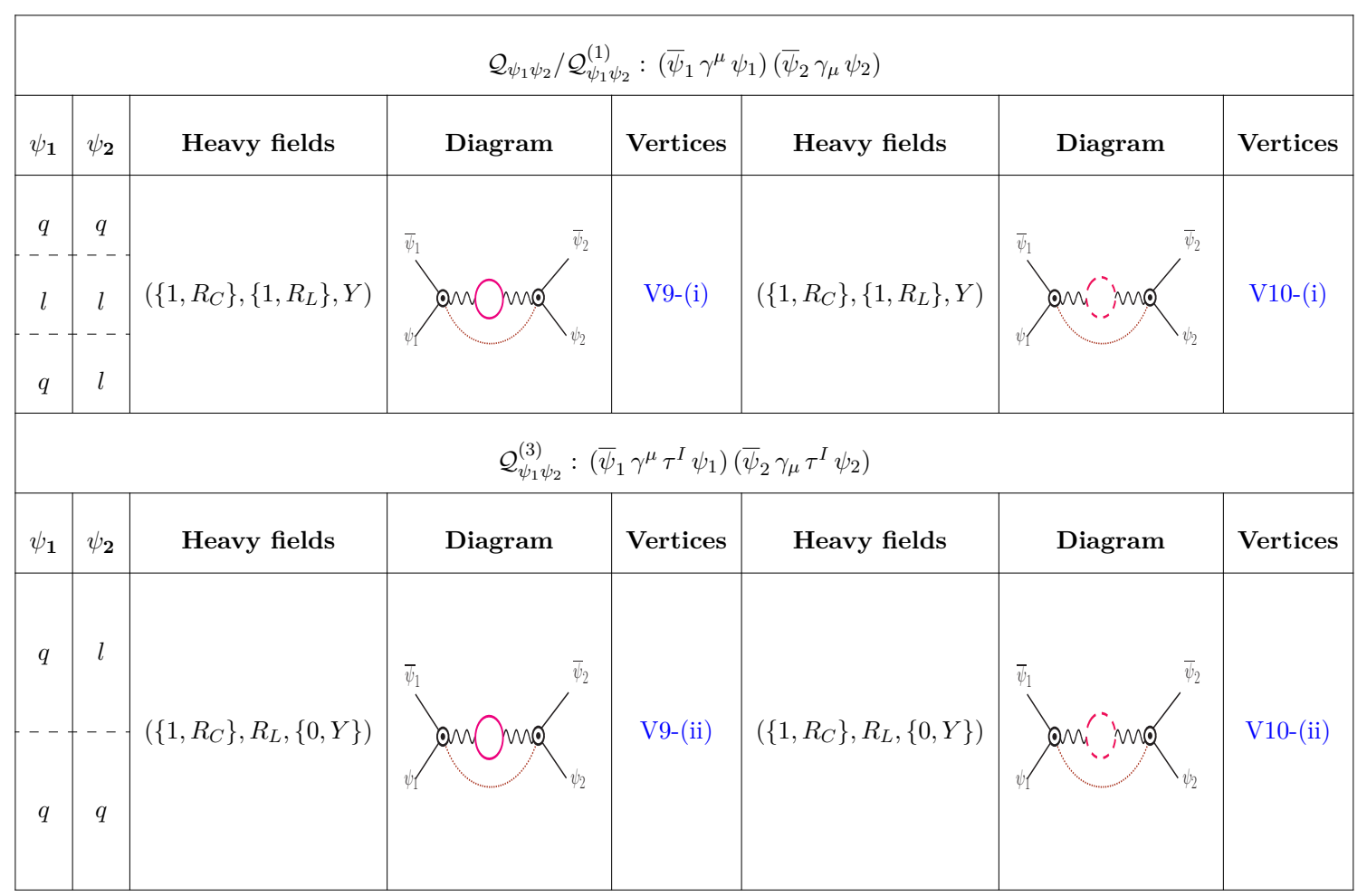

Table 12. Heavy field representations that are obtained by unfolding the $\psi^{4}$ operators, composed of the left chiral SM fermions $q$ and $l$, into non-trivial one-loop-level diagrams and the corresponding vertices. In each sub-table, operators with different fermions as external legs but leading to identical heavy field representations through similar diagrams have been grouped together. The headers schematically describe the covariant forms of the operators.

the form $\left(\bar{q}_{p} \gamma_{\mu} T^{A} q_{r}\right)\left(\bar{q}_{s} \gamma^{\mu} T^{A} q_{t}\right)$ and consequently the lack of a diagram involving $G_{\mu}^{A}$. Complete results for these operators have been presented in a condensed form in table 12 .

The form in which these operators appear within the SMEFT dimension-6 operator basis can only allow vector boson propagators in the unfolded diagrams but the "external states" corresponding to these same operators can also be obtained from tree-level processes involving a scalar propagator. The corresponding results have been highlighted in table 13 .

(ii) $(\boldsymbol{R} \overline{\boldsymbol{R}})(\boldsymbol{R} \overline{\boldsymbol{R}}): \quad$ this subclass contains operators constituted solely of the isospin singlet fermions $u, d, e$ and their conjugates, i.e., the operators $-\mathcal{Q}_{e e}, \mathcal{Q}_{u u}, \mathcal{Q}_{d d}, \mathcal{Q}_{e u}, \mathcal{Q}_{e d}, \mathcal{Q}_{u d}^{(1)}$, $\mathcal{Q}_{u d}^{(8)}$. These operators are also expressed as the Lorentz contraction of two 4 -vectors each of them being fermion bilinears of the form $\bar{\psi} \gamma_{\mu} \psi$. Therefore their unfolding incorporate the SM vector bosons and heavy fields appear in the form of scalar or fermion loops coupling to these vector bosons.

The diagrams corresponding to $\mathcal{Q}_{u d}^{(8)}$ incorporate $G_{\mu}^{A}$, while those for the rest of the operators incorporate $B_{\mu}$. Since all these fermions are isospin singlets, $W_{\mu}^{I}$ does not appear in any diagram. The results have been succinctly collected in table 14 .

Additionally, "the external states" corresponding to these same operators can also be obtained from tree-level processes involving a scalar propagator. The corresponding results have been collected in table 13 . 


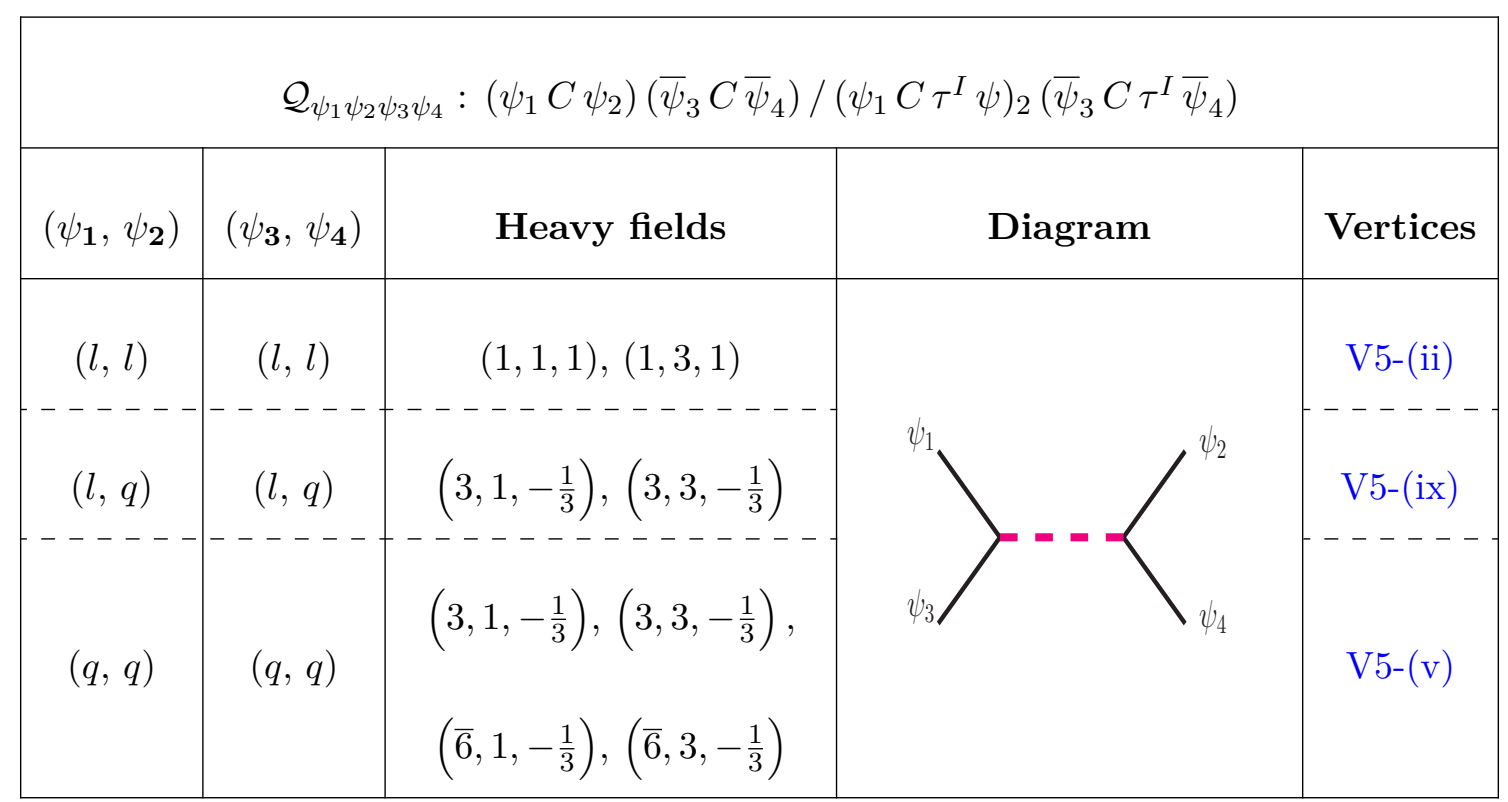

Table 13. Heavy field representations that are obtained by unfolding the $\psi^{4}$ operators, composed of the left chiral SM fermions $q$ and $l$, into a tree-level diagram yielding non-zero contributions and the corresponding vertices that constitute the diagram for individual cases. The operators have different fermions as external legs but they unfold through similar diagrams. The table header schematically describes the covariant forms of the operators.

(iii) $(\boldsymbol{L} \overline{\boldsymbol{L}})(\boldsymbol{R} \overline{\boldsymbol{R}}): \quad$ there are two different ways of expressing the operators of this subclass as products of fermion bilinears:

1. The first one is similar to the previous two cases with bilinears of the form $\bar{\psi} \gamma_{\mu} \psi$ and with the SM vector bosons in the diagrams. The diagrams corresponding to $\mathcal{Q}_{q u}^{(8)}$ and $\mathcal{Q}_{q d}^{(8)}$ incorporate $G_{\mu}^{A}$, while those for the rest of the operators incorporate $B_{\mu}$. Since in each operator two fermions are isospin singlets, $W_{\mu}^{I}$ does not appear in any diagram. Once again these diagrams have flavour conservation imposed at each vertex. The corresponding results are collected in table 16 .

2. For operators of this subclass, instead of forming fermion bilinears of the form $\bar{\psi} \gamma_{\mu} \psi$ which transform as 4 -vectors under Lorentz transformations, we can construct Lorentz scalars of the form $\bar{\psi}_{1} \psi_{2}$, where $\psi_{1}$ and $\psi_{2}$ have opposite chirality. As a result of this, in the unfolded diagram we can have the vertex $\bar{\psi}_{1} \psi_{2} \phi$ instead of $\bar{\psi} \gamma_{\mu} \psi V^{\mu}$. This brings into light a few interesting points:

- The presence of a Yukawa like vertex allows for the possibility of flavour violation.

- This rearrangement of external legs also allows the appearance of certain heavy scalars at the tree-level itself.

- Even though operators of the form $\left(\bar{\psi}_{1} \psi_{2}\right)\left(\bar{\psi}_{2} \psi_{1}\right)$ may appear to be unique with respect to those of the form $\left(\bar{\psi}_{1} \gamma_{\mu} \psi_{1}\right)\left(\bar{\psi}_{2} \gamma^{\mu} \psi_{2}\right)$, they are actually inter- 


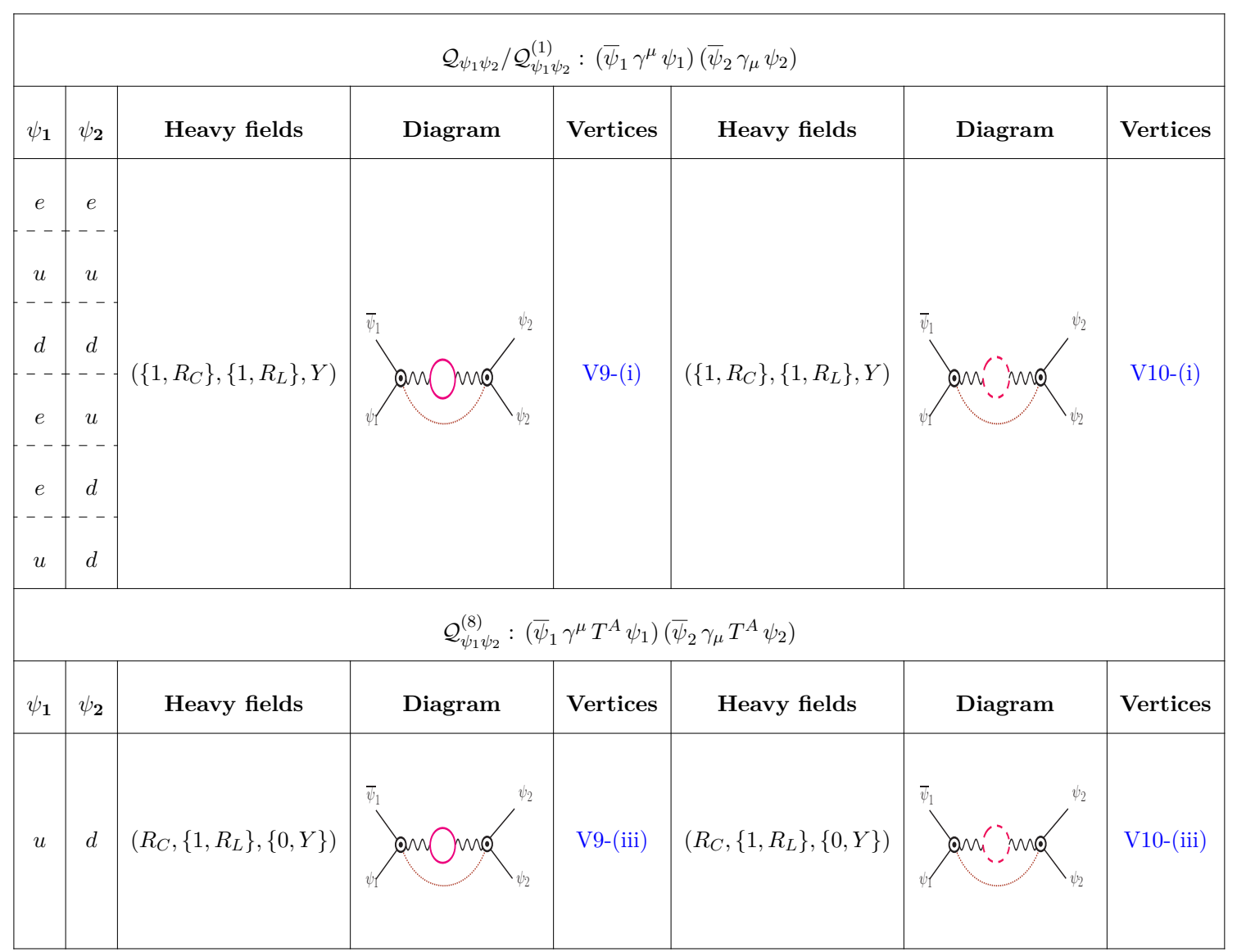

Table 14. Heavy field representations that are obtained by unfolding the $\psi^{4}$ operators, composed of the right chiral SM fermions $e, u$ and $d$, into non-trivial one-loop-level diagrams and the corresponding vertices. In each sub-table, operators with different fermions as external legs but leading to identical heavy field representations through similar diagrams have been grouped together. The headers schematically describe the covariant forms of the operators.

related through the Fierz relations of the $\gamma^{\mu}$-matrices or more appropriately ${ }^{5}$ the relations of the $\sigma^{\mu}\left(\equiv\left(\mathbf{1}_{2 \times 2}, \vec{\sigma}\right)\right)$ as shown below:

$$
\left(\sigma^{\mu}\right)_{\alpha \dot{\alpha}}\left(\sigma_{\mu}\right)_{\beta \dot{\beta}}=2 \epsilon_{\alpha \beta} \epsilon_{\dot{\alpha} \dot{\beta}},\left(\sigma^{\mu}\right)_{\alpha \dot{\alpha}}\left(\bar{\sigma}_{\mu}\right)^{\dot{\beta} \beta}=2 \delta_{\alpha}^{\beta} \delta_{\dot{\alpha}}^{\dot{\beta}},\left(\bar{\sigma}^{\mu}\right)^{\dot{\alpha} \alpha}\left(\bar{\sigma}_{\mu}\right)^{\dot{\beta} \beta}=2 \epsilon^{\alpha \beta} \epsilon^{\dot{\alpha} \dot{\beta}}
$$

Using these we can show, for instance:

$$
\begin{aligned}
\left(\bar{d} \gamma^{\mu} d\right)\left(\bar{q} \gamma_{\mu} q\right) & =\left(\bar{d}^{\alpha} \sigma_{\alpha \dot{\alpha}}^{\mu} d^{\dot{\alpha}}\right)\left(\bar{q}_{\dot{\beta}} \bar{\sigma}^{\mu \dot{\beta} \beta} q_{\beta}\right)=2\left(\bar{d}^{\alpha} q_{\beta} \bar{q}_{\dot{\beta}} d^{\dot{\alpha}}\right) \delta_{\alpha}^{\beta} \delta_{\dot{\alpha}}^{\dot{\beta}}=2(\bar{d} q)(\bar{q} d), \\
\left(\bar{d} \gamma^{\mu} T^{A} d\right)\left(\bar{q} \gamma_{\mu} T^{A} q\right) & =\left(\bar{d}^{\alpha} \sigma_{\alpha \dot{\alpha}}^{\mu} T^{A} d^{\dot{\alpha}}\right)\left(\bar{q}_{\dot{\beta}} T^{A} \bar{\sigma}^{\mu \dot{\beta} \beta} q_{\beta}\right)=2 \bar{d}^{\alpha} T^{A} q_{\beta} \bar{q}_{\dot{\beta}} T^{A} d^{\dot{\alpha}} \delta_{\alpha}^{\beta} \delta_{\dot{\alpha}}^{\dot{\beta}} \\
& =2\left(\bar{d} T^{A} q\right)\left(\bar{q} T^{A} d\right) .
\end{aligned}
$$

\footnotetext{
${ }^{5}$ It must be kept in mind that when we are speaking of $\sigma^{\mu}$ and $\gamma^{\mu}$ in the same sentence, it is implied that we are working in the Weyl basis, where $\gamma^{\mu}=\left(\frac{0}{\sigma} \sigma^{\mu}{ }_{0}^{\mu}\right)$ and for conveniently going between 2- and 4component notation of fermions, we can understand a 4-component left-chiral fermion as one with the last two entries 0. Similarly, a 4-component right chiral fermion would have its first two entries as 0 .
} 


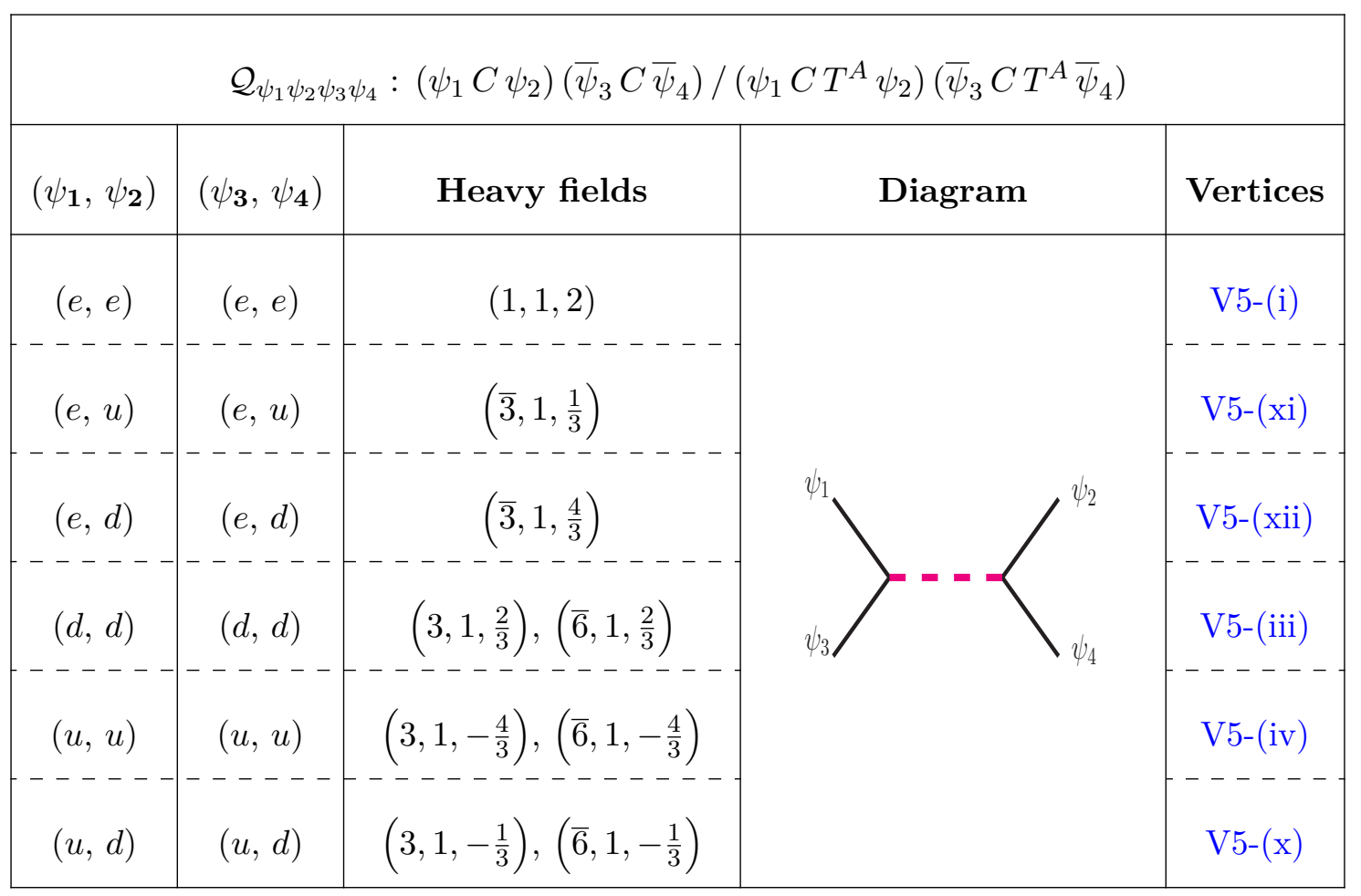

Table 15. Heavy field representations that are obtained by unfolding the $\psi^{4}$ operators, composed of the right chiral SM fermions $e, u$ and $d$, into a non-trivial tree-level diagram and the corresponding vertices for individual cases. The operators have different fermions as external legs but they unfold through similar diagrams. The table header schematically describes the covariant forms of the operators.

Hence, we can easily modify the form of the operators while staying within the confines of the complete and independent Warsaw basis [3].

In table 16, the heavy field representations obtained were common for the operators $\mathcal{Q}_{l e}, \mathcal{Q}_{l u}, \mathcal{Q}_{l d}, \mathcal{Q}_{q e}, \mathcal{Q}_{q u}^{(1)}$ and $\mathcal{Q}_{q d}^{(1)}$, and similarly for the operators $\mathcal{Q}_{q u}^{(8)}$ and $\mathcal{Q}_{q d}^{(8)}$ but after rearranging each operator in the manner suggested in eq. (3.4), we observe that there are heavy field representations which are common to some operators and other representations which are unique to certain operators. The specific results for each case have been catalogued in tables 17 and 18. In a minimal setting, the following diagrams appear for each case:

(a) Figure 8i which is a tree level diagram containing a heavy scalar propagator.

(b) Figure 8ii with light-heavy mixing in the loop involving a single heavy fermion propagator along with the SM scalar and an SM fermion.

(c) Figure 8ii with light-heavy mixing in the loop involving heavy scalar propagators and light SM fermion propagators.

(iv) $(\boldsymbol{L} \overline{\boldsymbol{R}})(\boldsymbol{R} \overline{\boldsymbol{L}}),(\overline{\boldsymbol{L}} \boldsymbol{R})(\overline{\boldsymbol{L}} \boldsymbol{R}):$ the first of these two subclasses contains a single operator $\mathcal{Q}_{\text {ledq }}$ and it allows heavy scalars with specific quantum numbers to appear through tree as well as loop diagrams. 


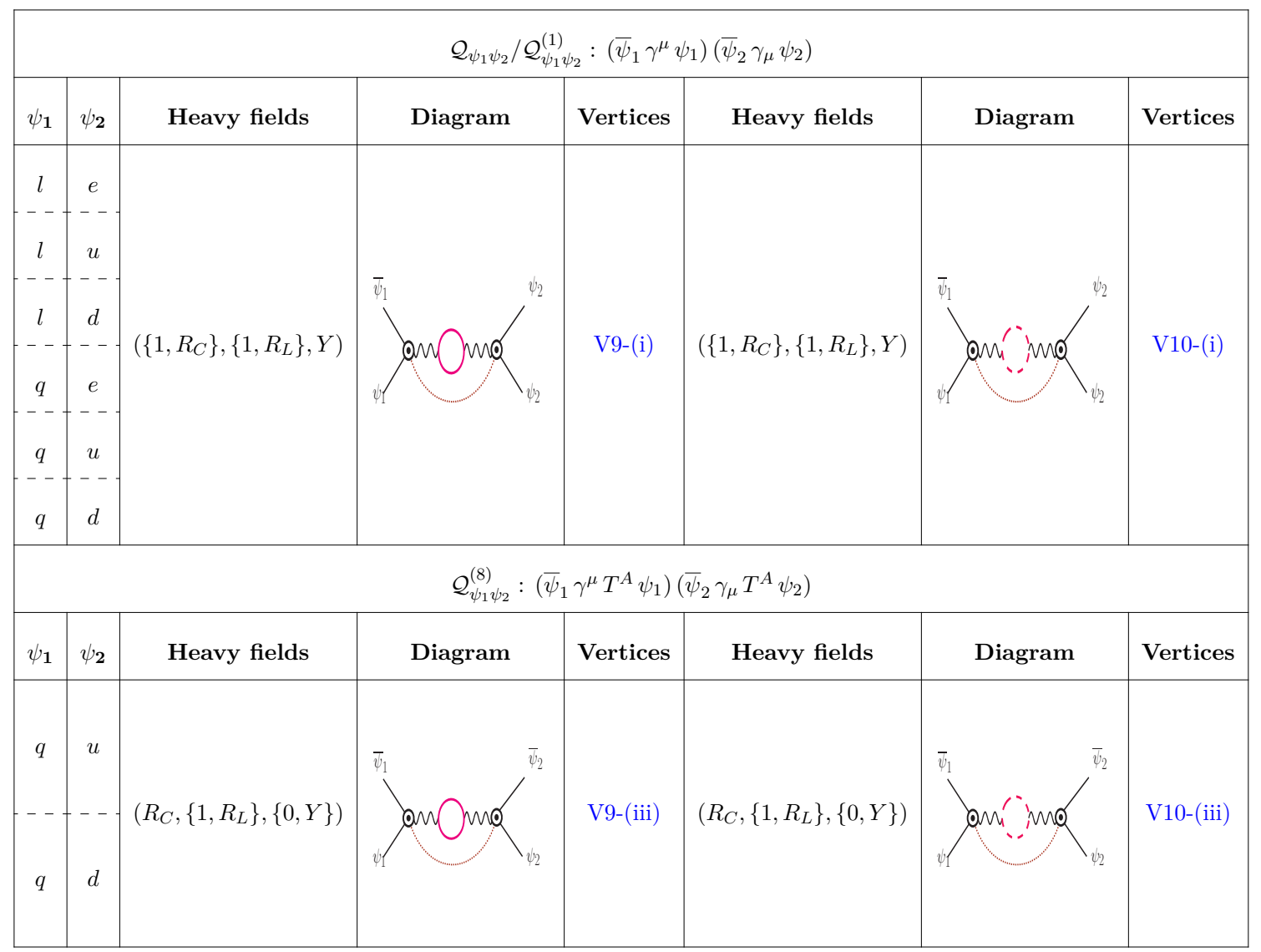

Table 16. Heavy field representations that are obtained by unfolding the $\psi^{4}$ operators, composed of both left and the right chiral SM fermions, into non-trivial one-loop-level diagrams and the corresponding vertices. Each of the processes highlighted here are flavour conserving. In each sub-table, operators with different fermions as external legs but leading to identical heavy field representations through similar diagrams have been grouped together. The headers schematically describe the covariant forms of the operators.

The other subclass contains four operators $\mathcal{Q}_{\text {quqd }}^{(1)}, \mathcal{Q}_{\text {quqd }}^{(8)}, \mathcal{Q}_{\text {lequ }}^{(1)}, \mathcal{Q}_{\text {lequ }}^{(3)}$. The first three of these admit heavy scalars through tree-level processes. Different representations are obtained by permuting the external legs suitably. $\mathcal{Q}_{\text {lequ }}^{(3)}$ is built of non-trivial tensor structures and therefore requires special attention. The results corresponding to $\mathcal{Q}_{\text {ledq }}, \mathcal{Q}_{\text {quqd }}^{(1)}$, $\mathcal{Q}_{\text {quqd }}^{(8)}$ and $\mathcal{Q}_{\text {lequ }}^{(1)}$ have been collected in table 19.

One can observe that $\mathcal{Q}_{\text {lequ }}^{(3)} \equiv\left(\bar{l}_{p}^{j} \sigma^{\mu \nu} e_{r}\right) \epsilon_{j k}\left(\bar{q}_{s}^{k} \sigma_{\mu \nu} u_{t}\right)$ contains the same external states as $\mathcal{Q}_{\text {lequ }}^{(1)} \equiv\left(\bar{l}_{p}^{j} e_{r}\right) \epsilon_{j k}\left(\bar{q}_{s}^{k} u_{t}\right)$ and the operator $\left(\bar{l}_{p}^{j} u_{r}\right) \epsilon_{j k}\left(\bar{q}_{s}^{k} e_{t}\right)$. Also, $\mathcal{Q}_{\text {lequ }}^{(3)}$ is related to these 2 operators as shown below:

$$
\begin{aligned}
\left(\bar{l}^{j} \sigma_{\mu \nu} e\right) \epsilon_{j k}\left(\bar{q}^{k} \sigma^{\mu \nu} u\right) & =\left[\left(\bar{l}^{j}\right)^{\alpha}\left(\sigma_{\mu \nu}\right)_{\alpha}^{\beta}(e)_{\beta}\right] \epsilon_{j k}\left[\left(\bar{q}^{k}\right)^{\rho}\left(\sigma^{\mu \nu}\right)_{\rho}^{\theta}(u)_{\theta}\right] \\
& =\left[\left(\bar{l}^{j}\right)^{\alpha}\left(\sigma_{\mu}\right)_{\alpha \dot{\beta}}\left(\bar{\sigma}_{\nu}\right)^{\dot{\beta} \beta}(e)_{\beta}\right] \epsilon_{j k}\left[\left(\bar{q}^{k}\right)^{\rho}\left(\sigma^{\mu}\right)_{\rho \dot{\theta}}\left(\bar{\sigma}^{\nu}\right)^{\dot{\theta} \theta}(u)_{\theta}\right] \\
& =4\left(\bar{l}^{j} e\right) \epsilon_{j k}\left(\bar{q}^{k} u\right)-8\left(\bar{l}^{j} u\right) \epsilon_{j k}\left(\bar{q}^{k} e\right) .
\end{aligned}
$$




\begin{tabular}{|c|c|c|c|c|c|}
\hline \multicolumn{3}{|c|}{$\mathcal{Q}_{l e}:\left(\bar{l}_{p} \gamma^{\mu} l_{r}\right)\left(\bar{e}_{s} \gamma_{\mu} e_{t}\right)$} & \multicolumn{3}{|c|}{$\mathcal{Q}_{l d}:\left(\bar{l}_{p} \gamma^{\mu} l_{r}\right)\left(\bar{d}_{s} \gamma_{\mu} d_{t}\right)$} \\
\hline Heavy fields & Diagram & Vertices & Heavy fields & Diagram & Vertices \\
\hline$\left(1,2, \frac{1}{2}\right)$ & & V5-(vi) & $\left(3,2, \frac{1}{6}\right)$ & & V5-(xvi) \\
\hline$(1,3,0),(1,1,0)$ & & V6-(i) & $(1,3,0),(1,1,0)$ & & V6-(i) \\
\hline$(1,3,1),(1,1,1)$ & & V6-(vi) & $(1,3,1),(1,1,1)$ & & V6-(vi) \\
\hline$\left(1,2, \frac{1}{2}\right)$ & & V6-(ii) & $\left(3,2, \frac{1}{6}\right)$ & & V6-(v) \\
\hline$\left(1,2, \frac{3}{2}\right)$ & & V6-(vii) & $\left(3,2,-\frac{5}{6}\right)$ & & V6-(x) \\
\hline$\left(3,2, \frac{7}{6}\right)$ & & V5-(xiii), V5-(xiv) & & & \\
\hline \multicolumn{3}{|c|}{$\mathcal{Q}_{l u}:\left(\bar{l}_{p} \gamma^{\mu} l_{r}\right)\left(\bar{u}_{s} \gamma_{\mu} u_{t}\right)$} & \multicolumn{3}{|c|}{$\mathcal{Q}_{q e}:\left(\bar{q}_{p} \gamma^{\mu} q_{r}\right)\left(\bar{e}_{s} \gamma_{\mu} e_{t}\right)$} \\
\hline Heavy fields & Diagram & Vertices & Heavy fields & Diagram & Vertices \\
\hline$\left(3,2, \frac{7}{6}\right)$ & & V5-(xiv) & $\left(3,2, \frac{7}{6}\right)$ & & V5-(xiv) \\
\hline$(1,3,0),(1,1,0)$ & & V6-(i) & $\left(3,1, \frac{2}{3}\right),\left(3,3, \frac{2}{3}\right)$ & & V6-(iii) \\
\hline$(1,3,1),(1,1,1)$ & & V6-(vi) & $\left(3,1,-\frac{1}{3}\right),\left(3,3,-\frac{1}{3}\right)$ & & V6-(viii) \\
\hline$\left(3,2, \frac{7}{6}\right)$ & & V6-(iv) & $\left(1,2, \frac{1}{2}\right)$ & & V6-(ii) \\
\hline$\left(3,2, \frac{1}{6}\right)$ & & V6-(ix) & $\left(1,2, \frac{3}{2}\right)$ & & V6-(vii) \\
\hline & & V5-(ix), V5-(x) & & & V5-(ix), V5-(xi) \\
\hline & & V5-(ix), V5-(xi) & & & V5-(v), V5-(xi) \\
\hline
\end{tabular}

Table 17. Heavy field representations that are obtained by unfolding the $\psi^{4}$ operators, composed of both left and right chiral SM fermions, into non-trivial tree- and (or) one-loop-level diagrams and the corresponding vertices. These diagrams are obtained after the form of the operators have been modified using Fierz identities. The processes highlighted here can allow for flavour violation. Each sub-table within the $2 \times 2$ grid corresponds to a different operator as mentioned in the respective headers. 


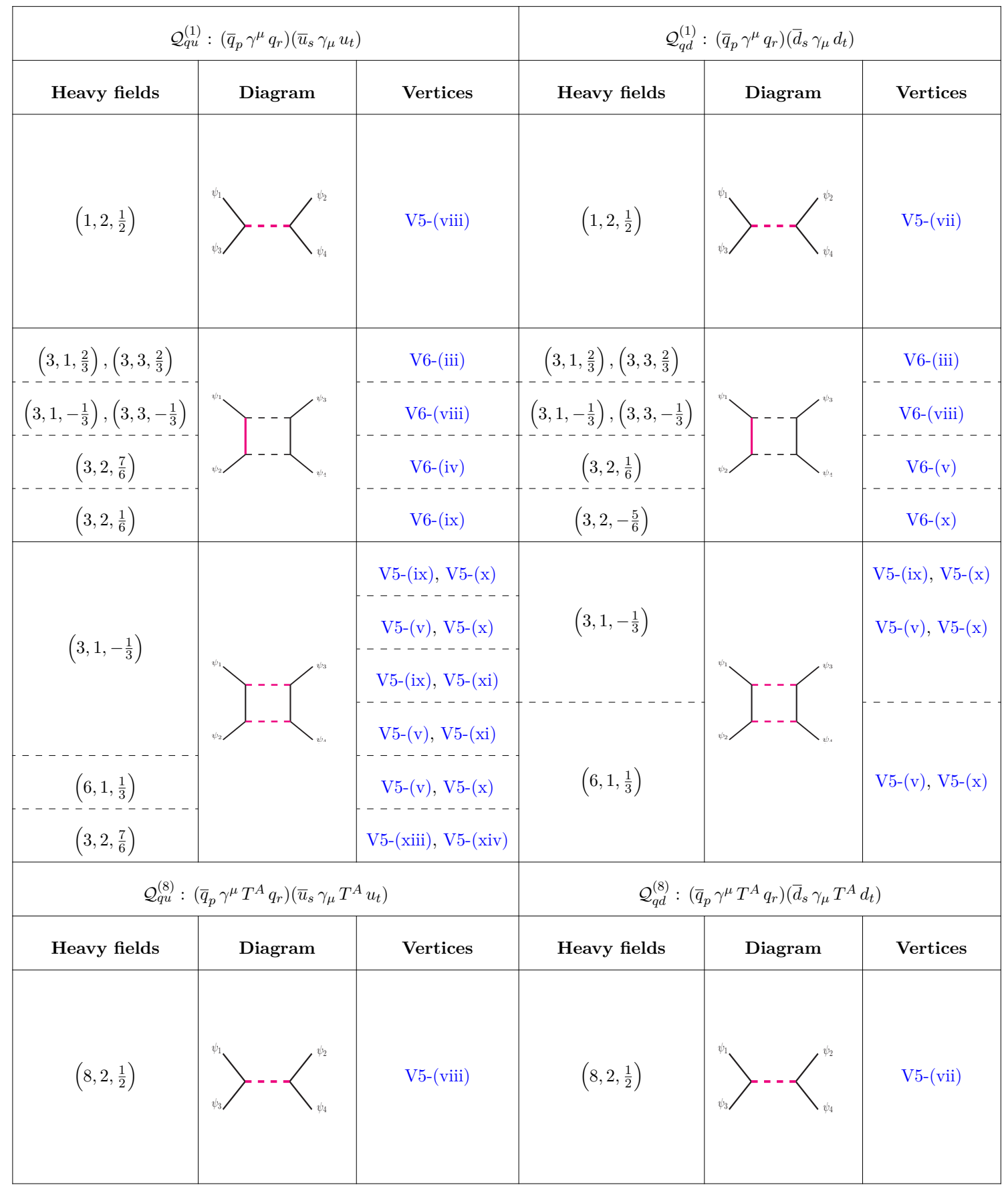

Table 18. Table 17 continued. A side by side comparison displays the similarities as well as the differences between the heavy field representations that can be obtained from operators that differ only with respect to the substitution of the up-quark for the down-quark. 


\begin{tabular}{|c|c|c|}
\hline \multicolumn{3}{|c|}{$\mathcal{Q}_{\text {ledq }}:\left(\bar{l}_{p}^{j} e_{r}\right)\left(\bar{d}_{s} q_{t j}\right)$} \\
\hline Heavy fields & Diagram & Vertices \\
\hline$\left(1,2, \frac{1}{2}\right)$ & & V5-(vi), V5-(vii) \\
\hline$\left(3,1,-\frac{1}{3}\right)$ & & V5-(v), V5-(ix), V5-(x), V5-(xi) \\
\hline \multicolumn{3}{|c|}{$\mathcal{Q}_{q u q d}^{(1)}:\left(\bar{q}_{p}^{j} u_{r}\right) \epsilon_{j k}\left(\bar{q}_{s}^{k} d_{t}\right)$} \\
\hline Heavy fields & Diagram & Vertices \\
\hline$\left(1,2, \frac{1}{2}\right)$ & & V5-(vii), V5-(viii) \\
\hline$\left(3,1,-\frac{1}{3}\right),\left(\overline{6}, 1,-\frac{1}{3}\right)$ & & V5-(v), V5-(x) \\
\hline \multicolumn{3}{|c|}{$\mathcal{Q}_{q u q d}^{(8)}:\left(\bar{q}_{p}^{j} T^{A} u_{r}\right) \epsilon_{j k}\left(\bar{q}_{s}^{k} T^{A} d_{t}\right)$} \\
\hline Heavy fields & Diagram & Vertices \\
\hline$\left(8,2, \frac{1}{2}\right)$ & & V5-(vii), V5-(viii) \\
\hline \multicolumn{3}{|c|}{$\mathcal{Q}_{\text {lequ }}^{(1)}:\left(\bar{l}_{p}^{j} e_{r}\right) \epsilon_{j k}\left(\bar{q}_{s}^{k} u_{t}\right) /\left(\bar{l}_{p}^{j} u_{r \alpha}\right) \epsilon_{j k}\left(\bar{q}_{s}^{k \alpha} e_{t}\right)$} \\
\hline Heavy fields & Diagram & Vertices \\
\hline$\left(1,2, \frac{1}{2}\right)$ & & V5-(vi), V5-(viii) \\
\hline$\left(3,1,-\frac{1}{3}\right)$ & & V5-(ix), V5-(xi) \\
\hline$\left(3,2, \frac{7}{6}\right)$ & & V5-(xiii), V5-(xiv) \\
\hline
\end{tabular}

Table 19. Heavy field representations that are obtained by unfolding the $\psi^{4}$ operators, composed of both left and right chiral SM fermions, into non-trivial tree- and (or) one-loop-level diagrams and the corresponding vertices. These operators contain atleast 3 unique fields as their constituents and they do not permit a vector boson propagators when we restrict to minimal scenarios. The operator highlighted in green color is constituted of the same external states as a SMEFT operator but leads to unique heavy field representations, see eq. (3.5). 


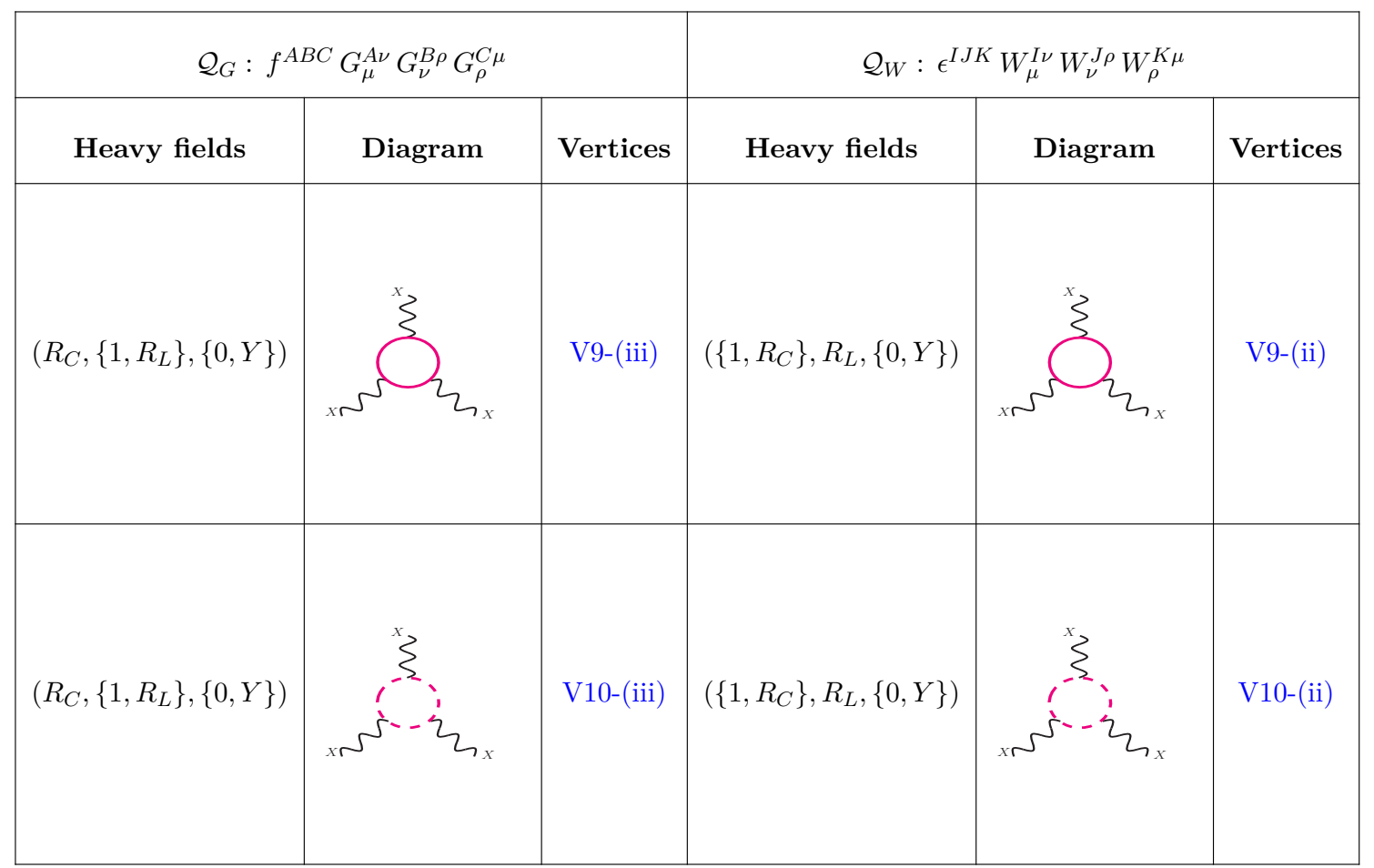

Table 20. Heavy field representations that are obtained by unfolding the $X^{3}$ operators into nontrivial one-loop-level diagrams and the corresponding vertices. A side by side comparison shows the subtle yet significant difference between the heavy field representations enveloped in these operators.

Here, we have used $\left(\sigma^{\mu \nu}\right)_{\alpha}^{\beta}=\left(\sigma^{\mu}\right)_{\alpha \dot{\beta}}\left(\bar{\sigma}^{\nu}\right)^{\dot{\beta} \beta},\left(\bar{\sigma}_{\mu \nu}\right)_{\dot{\alpha}}^{\dot{\beta}}=\left(\bar{\sigma}_{\mu}\right)^{\dot{\beta} \beta}\left(\sigma_{\nu}\right)_{\beta \dot{\alpha}}$ in addition to eq. (3.3). The last diagram in table 19 describes the unfolding of both $\mathcal{Q}_{\text {lequ }}^{(1)}$ and $\left(\bar{l}_{p}^{j} e_{r}\right) \epsilon_{j k}\left(\bar{q}_{s}^{k} u_{t}\right)$. To highlight this fact we have written the latter operator structure in colored text in table 19. Therefore, instead of unfolding $\mathcal{Q}_{\text {lequ }}^{(3)}$ explicitly, we can indirectly relate it to the heavy fields that lead to those two operators. However, if we choose to forego our conditions of minimality then, this operator can be unfolded into a multi-loop diagram with multiple heavy field propagators within some of the loops. This is discussed more properly in section 4.3.

\section{$3.7 \quad X^{3}$}

This class contains only two operators $-\mathcal{Q}_{G}$ and $\mathcal{Q}_{W}$. Heavy scalars, as well as heavy fermions, appear through one-loop-level diagrams, and the minimal choice corresponds to figures $9 \mathrm{i}$ and $9 \mathrm{v} . \mathcal{Q}_{G}$ only encapsulates color non-singlets and $\mathcal{Q}_{W}$ only isospin non-singlet representations, with no restrictions on the other quantum numbers in each case. These results have been summarized in table 20 .

\section{Departure from minimality}

While one can always study scenarios that depart radically from the notion of minimality described here, we have discussed a few cases that are necessary for the sake of completeness in the context of SMEFT. 


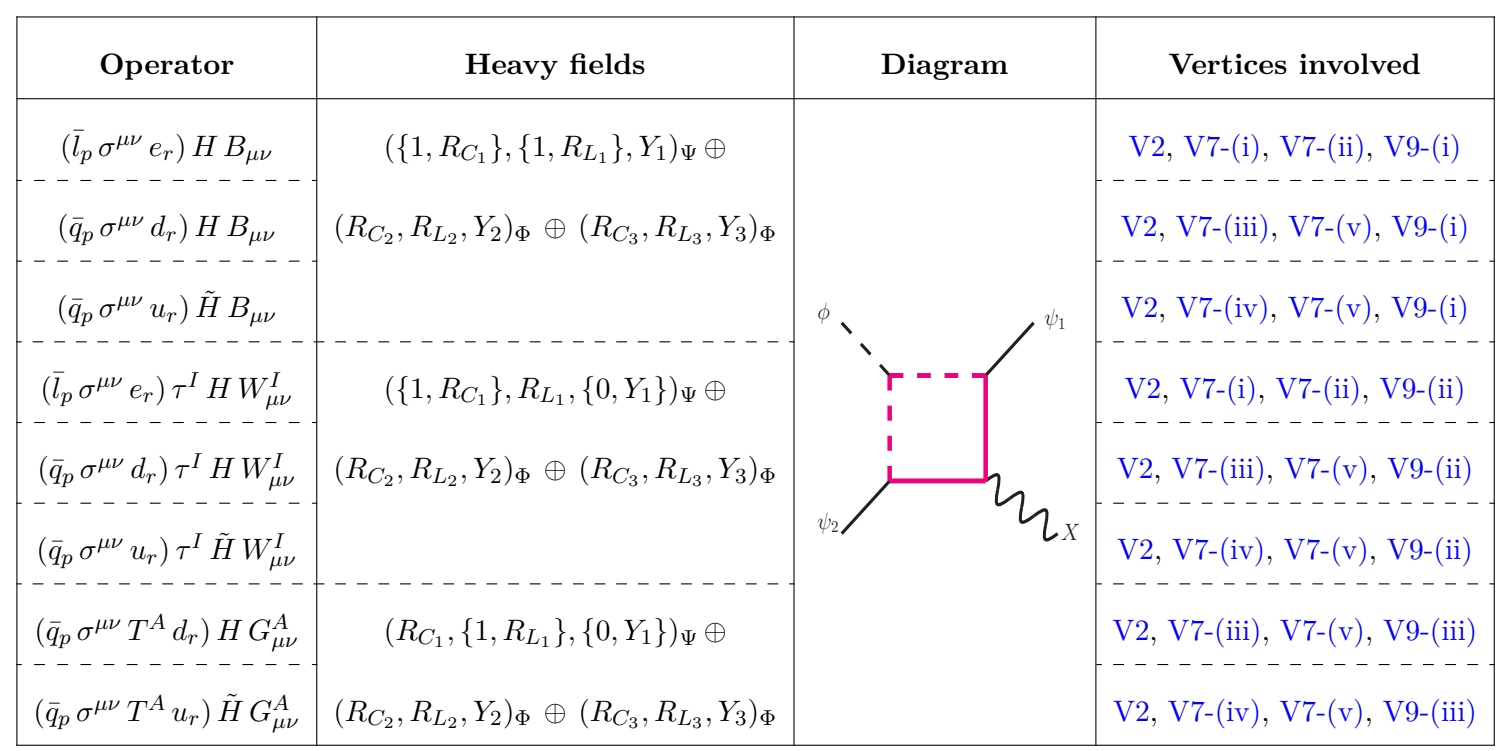

Table 21. Combinations of heavy field representations that are required to unfold the $\psi^{2} \phi X$ operators into non-trivial one-loop-level diagrams and the corresponding vertices for individual cases.

\subsection{Diagrams with multiple fundamental vertices}

As already discussed previously, the most straightforward departure from our notion of minimality occurs if we include diagrams such as figure 3ii, which includes both trilinear and quartic scalar vertices.

\subsection{Heavy-heavy mixing in the loops}

To depict the simultaneous appearance of multiple heavy fields in the loop, we consider operators of the $\psi^{2} \phi X$ class. For this class, no heavy field emerges through tree- or "simple" one-loop-level diagrams when the light fields are fixed to be the SM degrees of freedom. We have listed the general representations allowed for each operator in table 21. These must satisfy equations corresponding to the conservation of symmetries at each vertex.

\subsection{Multi-loop diagrams}

As discussed previously, to confine ourselves within a minimal analysis we can relate $\mathcal{Q}_{\text {lequ }}^{(3)}$ indirectly with the heavy fields embedded in the diagrams corresponding to $\mathcal{Q}_{\text {lequ }}^{(1)}$ and $\left(\bar{l}_{p}^{j} e_{r}\right) \epsilon_{j k}\left(\bar{q}_{s}^{k} u_{t}\right)$. But, if we relax the restriction of being minimal we can unfold $\mathcal{Q}_{\text {lequ }}^{(3)}$ into a three loop diagram having heavy-heavy-light mixing between two of the loops. The explicit combinations of heavy field representations for different permutations of the external legs have been described in table 22 . 


\begin{tabular}{|c|c|c|c|c|c|c|c|}
\hline \multicolumn{8}{|c|}{$\mathcal{Q}_{\text {lequ }}^{(3)}:\left(\bar{l}_{p}^{j} \sigma^{\mu \nu} e_{r}\right) \epsilon_{j k}\left(\bar{q}_{s}^{k} \sigma_{\mu \nu} u_{t}\right)$} \\
\hline$\left(\psi_{1}, \psi_{2}\right)$ & \multicolumn{2}{|c|}{ loop1 } & \multicolumn{2}{|c|}{ loop2 } & \multirow{2}{*}{$X$} & \multirow{2}{*}{ Corresponding diagram } & \multirow{2}{*}{ Vertices involved } \\
\hline$\left(\psi_{3}, \psi_{4}\right)$ & $\Phi_{1}$ & $\Psi_{1}$ & $\Phi_{2}$ & $\Psi_{2}$ & & & \\
\hline$(\bar{l}, e)$ & $\begin{array}{l}(1,1,0) \\
(1,3,0)\end{array}$ & $\left(1,2,-\frac{1}{2}\right)$ & $\begin{array}{l}(1,1,0) \\
(1,3,0)\end{array}$ & $\begin{array}{r}\left(3,1, \frac{2}{3}\right) \\
\left(3,3, \frac{2}{3}\right)\end{array}$ & $\begin{array}{c}B \\
--- \\
B / W\end{array}$ & & V1-(ii), V6-(ii), V6-(iii) \\
\hline$(\bar{q}, u)$ & $\begin{array}{l}(1,1,1) \\
(1,3,1)\end{array}$ & $\left(1,2, \frac{3}{2}\right)$ & $\begin{array}{l}(1,1,1) \\
(1,3,1)\end{array}$ & $\begin{array}{r}\left(\overline{3}, 1, \frac{1}{3}\right) \\
\left(\overline{3}, 3, \frac{1}{3}\right)\end{array}$ & $\begin{array}{c}B \\
--- \\
B / W\end{array}$ & I & V1-(i), V6-(vii), V6-(viii) \\
\hline$(e, \bar{l})$ & $\begin{array}{l}(1,1,0) \\
-\end{array}$ & $(1,1,1)$ & $(1,1,0)$ & $\left(\overline{3}, 2, \frac{1}{6}\right)$ & $\begin{array}{c}B / W \\
---\end{array}$ & ---- & V1-(ii), V6-(vi), V6-(ix) \\
\hline$(u, \bar{q})$ & $(1,3,1)$ & $(1,3,0)$ & $\begin{array}{l}(1,1,1) \\
(1,3,1)\end{array}$ & $\left(3,2, \frac{5}{6}\right)$ & $W$ & & V1-(i), V6-(i), V6-(iv) \\
\hline
\end{tabular}

Table 22. Combinations of heavy field representations that appear within the loops when the operator $\mathcal{Q}_{\text {lequ }}^{(3)}$ is unfolded into a multi-loop diagram. The different orientations of the external legs and the choice of the intermediate vector boson have been considered separately and the vertices have been listed for individual cases.

\begin{tabular}{|c|c|c|c|c|c|c|c|}
\hline Operator & Table & Operator & Table & Operator & Table & Operator & Table \\
\hline $\mathcal{Q}_{H}$ & 5 & $\mathcal{Q}_{H \square}, \mathcal{Q}_{H \mathcal{D}}$ & 6 & $\mathcal{Q}_{e H}, \mathcal{Q}_{u H}, \mathcal{Q}_{d H}$ & 8 & $\mathcal{Q}_{H W B}$ & 11 \\
\hline $\mathcal{Q}_{H l}^{(1)}, \mathcal{Q}_{H e}, \mathcal{Q}_{H l}^{(3)}$, & 9 & $\begin{array}{c}\mathcal{Q}_{q q}^{(1)}, \mathcal{Q}_{l q}^{(1)}, \mathcal{Q}_{l l} \\
\mathcal{Q}_{l q}^{(3)}, \mathcal{Q}_{q q}^{(3)}\end{array}$ & 12 & $\mathcal{Q}_{e e}, \mathcal{Q}_{u u}, \mathcal{Q}_{d d}$ & 14 & $\mathcal{Q}_{l e}, \mathcal{Q}_{l u}, \mathcal{Q}_{q e}$, & 16 \\
$\mathcal{Q}_{H q}^{(1)}, \mathcal{Q}_{H u}, \mathcal{Q}_{H d}, \mathcal{Q}_{H q}^{(3)}$ & & $\mathcal{Q}_{e u}, \mathcal{Q}_{e d}, \mathcal{Q}_{u d}^{(1)}, \mathcal{Q}_{u d}^{(8)}$ & & $\mathcal{Q}_{q u}^{(1)}, \mathcal{Q}_{q u}^{(8)}, \mathcal{Q}_{q d}^{(1)}, \mathcal{Q}_{q d}^{(8)}$ & \\
\hline $\mathcal{Q}_{G}, \mathcal{Q}_{W}$ & 20 & $\mathcal{Q}_{l d}$ & 18 & $\mathcal{Q}_{H G}, \mathcal{Q}_{H W}, \mathcal{Q}_{H B}$ & 10 & & \\
\hline
\end{tabular}

Table 23. List of dimension-6 SMEFT operators which receive non-zero contributions from the scalar lepto-quark $\left(3,2, \frac{1}{6}\right)$ based on the diagrammatic unfolding of the operators. Also listed are the tables where the unfolding has explicitly been demonstrated for each operator.

\section{Operator driven BSM construction: validation and illustration through examples}

\subsection{Validating the diagrammatic method}

The analysis thus far has centred on unfolding the dimension-6 effective operators of SMEFT diagrammatically and obtaining BSM fields that can offer non-zero contributions to them. Here, we have demonstrated the consistency of our results with those of a topdown analysis. Focussing on a single heavy scalar, here the lepto-quark $\Theta$ with quantum numbers $\left(3,2, \frac{1}{6}\right)$, we have first collected the effective operators that have been found to receive contributions from it based on our diagrammatic approach, in table 23.

To test the validity of our method and our results, we first build the most general UV Lagrangian for a model where the SM field content is extended by a lepto-quark scalar $\Theta$ 
with gauge quantum numbers $\left(3,2, \frac{1}{6}\right)$ under the SM gauge groups using GrIP [7], which is shown in the covariant form below:

$$
\begin{aligned}
\mathcal{L}_{\Theta}= & \mathcal{L}_{\mathrm{SM}}+\left|\mathcal{D}_{\mu} \Theta\right|^{2}-m_{\Theta}^{2}|\Theta|^{2}-\eta_{1}\left(H^{\dagger} H\right)\left(\Theta^{\dagger} \Theta\right)-\eta_{2}\left(\Theta^{\dagger} \tau^{I} \Theta\right)\left(H^{\dagger} \tau^{I} H\right) \\
& -\lambda_{1}\left(\Theta^{\dagger} \Theta\right)^{2}-\lambda_{2}\left(\Theta^{\dagger} \tau^{I} \Theta\right)^{2}-\left(\epsilon_{i j} y_{\Theta}^{p r} \Theta^{\alpha i} \bar{d}_{p \alpha} l_{r}^{j}+\text { h.c. }\right) .
\end{aligned}
$$

Here, $\mathcal{L}_{\mathrm{SM}}$ represents the Standard Model Lagrangian. We have used CoDEx [28] to integrate-out the heavy scalar $\Theta$ and determine the SMEFT dimension-6 effective operators. The output from CoDEx exhibit non-zero Wilson coefficients for the following operators at the matching scale:

$$
\begin{aligned}
& \mathcal{Q}_{H D}, \mathcal{Q}_{l l}, \mathcal{Q}_{H u}, \mathcal{Q}_{H d}, \mathcal{Q}_{H e}, \mathcal{Q}_{H q}^{(1)}, \mathcal{Q}_{H l}^{(1)}, \mathcal{Q}_{H l}^{(3)}, \mathcal{Q}_{H q}^{(3)}, \mathcal{Q}_{H W B}, \mathcal{Q}_{H \square}, \mathcal{Q}_{H B}, \mathcal{Q}_{H W}, \mathcal{Q}_{H}, \mathcal{Q}_{G}, \mathcal{Q}_{H G}, \\
& \mathcal{Q}_{e H}, \mathcal{Q}_{u H}, \mathcal{Q}_{d H}, \mathcal{Q}_{q q}^{(1)}, \mathcal{Q}_{q q}^{(3)}, \mathcal{Q}_{u u}, \mathcal{Q}_{d d}, \mathcal{Q}_{u d}^{(1)}, \mathcal{Q}_{l q}^{(1)}, \mathcal{Q}_{e e}, \mathcal{Q}_{e u}, \mathcal{Q}_{e d}, \mathcal{Q}_{l e}, \mathcal{Q}_{l u}, \mathcal{Q}_{l d}, \mathcal{Q}_{q e}, \mathcal{Q}_{q u}^{(1)}, \mathcal{Q}_{q d}^{(1)}, \\
& \mathcal{Q}_{l q}^{(3)}, \mathcal{Q}_{W}, \mathcal{Q}_{u d}^{(8)}, \mathcal{Q}_{q d}^{(8)}, \mathcal{Q}_{q u}^{(8)} .
\end{aligned}
$$

These operators are completely in agreement with the results of table 23 . This shows that results obtained using the techniques outlined in this paper are consistent with the results available in the literature $[41,42,51]$.

\subsection{The minimal extension of the SM as root of CP even D6 SMEFT operators}

As the first application of our method, we have discussed how one can extend the SM degrees of freedom in the most minimal way so as to explain the origin of as many dimension- 6 $C P$ and $B-, L$ - conserving operators as possible. Out of the 59 operators $^{6}$ that constitute a complete and independent basis for SMEFT at dimension- 6 only 52 are found to be $C P$ conserving.

Since our aim is to build minimal extensions of the SM, based on the list of heavy field representations that have already been tabulated corresponding to every single operator, one must first identify the representations which are common to the largest subset of operators. We have found that the scalar field $\Theta_{\left(3,2, \frac{1}{6}\right)}$ provides non-zero contributions to 39 operators, while $\mathcal{H}_{\left(1,2, \frac{1}{2}\right)}$ (which constitutes the Two Higgs Doublet Model [61-63]) leads to 37 operators. The latter of the two is more minimal on account of it being an SU(3) singlet. Therefore we include it as the first extension to our degrees of freedom. Next, one can see that the operators involving gluons $-\mathcal{Q}_{G}, \mathcal{Q}_{H G}$ and those that are products of fermion bilinears transforming as $\mathrm{SU}(3)$ octets $-\mathcal{Q}_{u d}^{(8)}, \mathcal{Q}_{q d}^{(8)}, \mathcal{Q}_{q u}^{(8)}$ can only be generated by scalars with non-trivial SU(3) quantum numbers. This motivates us to include a second heavy field. We have chosen $\varphi_{\left(3,1,-\frac{1}{3}\right)}$ which being a color triplet $\mathrm{SU}(2)$ singlet so as to ensure minimality.

In table 24, we have listed all 42 operators that receive non-zero contributions from a color-singlet, isospin-doublet scalar $\mathcal{H}$ and (or) a color-triplet, isospin-singlet scalar $\varphi$. The entries in black colour are only generated by $\mathcal{H}$, those in pink are generated by $\varphi$ alone, while the ones in green receive contributions from both these fields. For easy reference, adjacent to each operator (or a collection of operators) we have also specified the table where it has been shown how unfolding the operator yields $\mathcal{H}$ and (or) $\varphi$.

\footnotetext{
${ }^{6}$ All these conserve baryon and lepton numbers.
} 


\begin{tabular}{|c|c|c|c|c|c|c|c|}
\hline Operator & Table & Operator & Table & Operator & Table & Operator & Table \\
\hline $\mathcal{Q}_{H}$ & 5 & $\mathcal{Q}_{H \square}, \mathcal{Q}_{H \mathcal{D}}$ & 6 & $\mathcal{Q}_{e H}, \mathcal{Q}_{u H}, \mathcal{Q}_{d H}$ & 7 & $\mathcal{Q}_{q u q d}^{(1)}, \mathcal{Q}_{\text {lequ }}^{(1)}, \mathcal{Q}_{\text {ledq }}$ & 19 \\
\hline $\begin{array}{c}\mathcal{Q}_{H l}^{(1)}, \mathcal{Q}_{H e}, \mathcal{Q}_{H l}^{(3)}, \\
\mathcal{Q}_{H q}^{(1)}, \mathcal{Q}_{H u}, \mathcal{Q}_{H d}, \mathcal{Q}_{H q}^{(3)}\end{array}$ & 9 & $\begin{array}{c}\mathcal{Q}_{q q}^{(1)}, \mathcal{Q}_{l q}^{(1)}, \mathcal{Q}_{l l} \\
\mathcal{Q}_{l q}^{(3)}, \mathcal{Q}_{q q}^{(3)}\end{array}$ & 12 & $\begin{array}{c}\mathcal{Q}_{e e}, \mathcal{Q}_{u u}, \mathcal{Q}_{d d} \\
\mathcal{Q}_{e u}, \mathcal{Q}_{e d}, \mathcal{Q}_{u d}^{(1)}, \mathcal{Q}_{u d}^{(8)}\end{array}$ & 14 & $\begin{array}{c}\mathcal{Q}_{l e}, \mathcal{Q}_{l u}, \mathcal{Q}_{q e} \\
\mathcal{Q}_{q u}^{(1)}, \mathcal{Q}_{q d}^{(1)}, \mathcal{Q}_{q d}^{(8)}, \mathcal{Q}_{q u}^{(8)}\end{array}$ & 16 \\
\hline $\mathcal{Q}_{W}, \mathcal{Q}_{G}$ & 20 & $\mathcal{Q}_{l d}$ & 16,17 & $\mathcal{Q}_{H W}, \mathcal{Q}_{H B}, \mathcal{Q}_{H G}$ & 10 & $\mathcal{Q}_{H W B}$ & 11 \\
\hline
\end{tabular}

Table 24. SMEFT dimension-6 effective operators unfolded by minimal extension. The operators in black are generated by the $\mathcal{H}_{\left(1,2, \frac{1}{2}\right)}$ extension alone. Further extension by the $\varphi_{\left(3,1,-\frac{1}{3}\right)}$ generates the extra operators highlighted in pink color. The ones shown in green are common to both these new fields.

Of the remaining 10 operators, 8 belong to the $\psi^{2} \phi X$ class and these can only be generated using models containing both scalar and fermion extensions, i.e., we are required to forego the notion of minimality, see table 21, as long as one only considers 1-particleirreducible (1PI) diagrams. The last two operators are $\mathcal{Q}_{q u q d}^{(8)}, \mathcal{Q}_{\text {lequ }}^{(3)}$ which belong to the $\psi^{4}$ class and require special attention. The first of these $\mathcal{Q}_{q u q d}^{(8)}$ can be directly generated by only a color-octet scalar extension or indirectly by a color-sextet scalar if one takes Fierz identities into account. Similarly, $\mathcal{Q}_{\text {lequ }}^{(3)}$ can be shown to receive contributions from $\mathcal{H}$ and $\varphi$ after employing certain Fierz identities, see eq. (3.5) and table 19. But its unfolding can only be conducted at the level of multi-loop diagrams, see table 22 .

\subsection{Role of observables on the choice of BSMs}

The analyses based on SMEFT start with the construction of a UV Lagrangian, followed by integrating out heavy fields to obtain effective operators and ultimately connecting those operators to well-defined observables. The prevalence of a large number of candidate scenarios that appear to be distinct makes conducting comparative analyses and verifying claims of one model being more vital than others, an arduous task.

This is where our techniques truly shine. Based on the particular observable we are interested in, we can readily obtain the set of relevant operators. Then, using the results of this paper as a dictionary, i.e., by looking at the unfolding of these operators catalogued here, one can easily arrive at the list of heavy field representations which are actually relevant for such observables. To illustrate this point, we have considered the operators contributing to the electroweak precision observables at leading order (EWPO-LO) [43], listed below:

$$
\left\{\mathcal{Q}_{l l}, \mathcal{Q}_{H \mathcal{D}}, \mathcal{Q}_{H W B}, \mathcal{Q}_{H q}^{(3)}, \mathcal{Q}_{H l}^{(3)}, \mathcal{Q}_{H q}^{(1)}, \mathcal{Q}_{H l}^{(1)}, \mathcal{Q}_{H e}, \mathcal{Q}_{H u}, \mathcal{Q}_{H d}\right\}
$$

We have followed two separate routes, first, where the SM extension is a scalar and second where it is a fermion. The procedure has been elucidated in table 25 .

For the case of scalar extensions, the operator $\mathcal{Q}_{H \mathcal{D}}$ allows all possible representations (trivial as well as non-trivial). Taking the operators $\left\{\mathcal{Q}_{l l}, \mathcal{Q}_{H q}^{(1)}, \mathcal{Q}_{H l}^{(1)}, \mathcal{Q}_{H e}, \mathcal{Q}_{H u}, \mathcal{Q}_{H d}\right\}$ into 


\begin{tabular}{|c|c|c|}
\hline \multicolumn{3}{|c|}{ Scalar extension } \\
\hline Operators & Heavy Fields & Table \\
\hline$\left\{\mathcal{Q}_{H \mathcal{D}}\right\}$ & $\left(\left\{1, R_{C}\right\},\left\{1, R_{L}\right\},\{0, Y\}\right)$ & 6 \\
+ & $\downarrow$ & \\
$\left\{\mathcal{Q}_{l l}, \mathcal{Q}_{H q}^{(1)}, \mathcal{Q}_{H l}^{(1)}, \mathcal{Q}_{H e}, \mathcal{Q}_{H u}, \mathcal{Q}_{H d}\right\}$ & $\left(\left\{1, R_{C}\right\},\left\{1, R_{L}\right\}, Y\right)$ & 9,12 \\
+ & $\downarrow$ & \\
$\left\{\mathcal{Q}_{H q}^{(3)}, \mathcal{Q}_{H l}^{(3)}, \mathcal{Q}_{H W B}\right\}$ & $\left(\left\{1, R_{C}\right\}, R_{L}, Y\right)$ & 9,11 \\
\hline
\end{tabular}

\begin{tabular}{|c|c|c|}
\hline \multicolumn{3}{|c|}{ Fermion extension } \\
\hline Operators & Heavy Fields & Table \\
\hline$\left\{\mathcal{Q}_{l l}, \mathcal{Q}_{H q}^{(1)}, \mathcal{Q}_{H l}^{(1)}, \mathcal{Q}_{H e}, \mathcal{Q}_{H u}, \mathcal{Q}_{H d}\right\}$ & $\left(\left\{1, R_{C}\right\},\left\{1, R_{L}\right\}, Y\right)$ & 9,12 \\
\hline+ & $\downarrow$ & \\
\hline$\left\{\mathcal{Q}_{H q}^{(3)}, \mathcal{Q}_{H l}^{(3)}\right\}$ & $\left(\left\{1, R_{C}\right\}, R_{L}, Y\right)$ & 9 \\
\hline+ & $\downarrow$ & \\
\hline$\left\{\mathcal{Q}_{H W B}\right\}$ & $\begin{array}{c}\left(R_{C}, R_{L}, Y\right) \in\left\{\left(3,3,-\frac{1}{3}\right),\left(3,3, \frac{2}{3}\right),\left(1,2, \frac{3}{2}\right),\left(1,2, \frac{1}{2}\right)\right. \\
\left.(1,3,1),\left(3,2, \frac{7}{6}\right),\left(3,2, \frac{1}{6}\right),\left(3,2,-\frac{5}{6}\right)\right\}\end{array}$ & 11 \\
\hline+ & $\downarrow$ & \\
\hline$\left\{\mathcal{Q}_{H \mathcal{D}}\right\}$ & $\begin{array}{l}\text { Necessitates the inclusion of a second heavy fermion }\left(R_{C^{\prime}}, R_{L^{\prime}}, Y^{\prime}\right) \\
\text { such that }\left(R_{C}, R_{L}, Y\right) \oplus\left(R_{C^{\prime}}, R_{L^{\prime}}, Y^{\prime}\right)=\left(1,2, \pm \frac{1}{2}\right) \text {, see V8 }\end{array}$ & 6 (last entry) \\
\hline
\end{tabular}

Table 25. Restrictions imposed on the heavy field representation as we take into account more and more operators relevant to electroweak precision observables. The "+" sign indicates that more operators are being added and " $\downarrow$ " shows how the permitted representations get modified gradually. For each step we have also referred to the tables where the relevant diagrams have been catalogued. 
account it is evident that only fields with non-zero hypercharge can be included. Finally, bringing $\mathcal{Q}_{H W B}, \mathcal{Q}_{H q}^{(3)}$, and $\mathcal{Q}_{H l}^{(3)}$ into the picture we get another constraint that the $\mathrm{SU}(2)$ quantum number must also be non-singlet. For the fermion case the procedure takes a different shape. Starting with $\left\{\mathcal{Q}_{l l}, \mathcal{Q}_{H q}^{(1)}, \mathcal{Q}_{H l}^{(1)}, \mathcal{Q}_{H e}, \mathcal{Q}_{H u}, \mathcal{Q}_{H d}\right\}$, restriction is set on the $\mathrm{U}(1)$ charge and including $\mathcal{Q}_{H q}^{(3)}, \mathcal{Q}_{H l}^{(3)}$ constrains the $\mathrm{SU}(2)$ quantum number. Then, adding $\mathcal{Q}_{H W B}$ we get well-defined heavy fermion representations as shown in table 25. Lastly, adding $\mathcal{Q}_{H \mathcal{D}}$ to the list suggests that we require more than a single fermion extension of the SM corresponding to each of the eight representations listed before.

Thus we have seen how, by progressively enlarging the operator set, we can be led to well-defined heavy field representations that actually contribute to the observables under consideration.

\section{Conclusion and remarks}

In this work, we have highlighted the principles and salient features of an operator driven prescription for UV model building. Starting from the bottom-up extension of a low energy theory, we have described how to catalogue heavy field representations that provide nonzero contributions to specific processes and observables of the low-energy theory. The main procedure involves the identification of Lorentz invariant renormalizable vertices followed by fixing some of the legs of those vertices to be the low energy quantum fields and investigating the heavy field representations (or their combinations in some cases) which can be assigned to the remaining leg(s) while ensuring the conservation of the internal symmetries of the theory. Thereafter, it has been described how the higher mass dimension effective operators of the low energy theory can be "unfolded" into tree- and loop-level processes using these vertices involving light as well as heavy fields.

Following a general discussion using schematic diagrams, we have made our ideas concrete by using the example of the Standard Model as the low energy theory and by exhibiting how to unfold each operator belonging to the dimension- 6 basis of SMEFT. We have kept our analysis minimal by only considering such UV theories that do not extend the SM gauge group. Also, the notion of minimality is reflected in the choice of tree and one-level diagrams included for specific cases. We have briefly described how non-minimal scenarios can be approached. Using appropriate examples, we have demonstrated how our results agree with those obtained using the conventional top-down approach of EFT.

The discussion underlines the economy as well as the systematic nature of our approach where the most significant heavy field extension(s) can be arrived at by examining the effective operators of the low energy theory alone. This is in contrast to the strenuous endeavour of building numerous plausible UV Lagrangians, integrating out the heavy fields to obtain subsets of the low energy effective Lagrangian and conducting multiple comparative analyses to adjudge the significance of the candidate UV models.

\section{Acknowledgments}

The work of JC, SDB and SUR is supported by the Science and Engineering Research Board, Government of India, under the agreements SERB/PHY/2016348 (Early Career 


\begin{tabular}{|c|c|c|c|c|c|c|}
\hline Field & $\mathrm{SU}(3)_{C}$ & $\mathrm{SU}(2)_{L}$ & $\mathrm{U}(1)_{Y}$ & Baryon No. & Lepton No. & Spin \\
\hline$H$ & 1 & 2 & $1 / 2$ & 0 & 0 & 0 \\
$q_{L}^{p}$ & 3 & 2 & $1 / 6$ & $1 / 3$ & 0 & $1 / 2$ \\
$u_{R}^{p}$ & 3 & 1 & $2 / 3$ & $1 / 3$ & 0 & $1 / 2$ \\
$d_{R}^{p}$ & 3 & 1 & $-1 / 3$ & $1 / 3$ & 0 & $1 / 2$ \\
$l_{L}^{p}$ & 1 & 2 & $-1 / 2$ & 0 & -1 & $1 / 2$ \\
$e_{R}^{p}$ & 1 & 1 & -1 & 0 & -1 & $1 / 2$ \\
\hline$G_{\mu}^{A}$ & 8 & 1 & 0 & 0 & 0 & 1 \\
$W_{\mu}^{I}$ & 1 & 3 & 0 & 0 & 0 & 1 \\
$B_{\mu}$ & 1 & 1 & 0 & 0 & 0 & 1 \\
\hline
\end{tabular}

Table 26. Standard Model: gauge and global quantum numbers and spins of the fields. Here, $A=1,2, \cdots, 8 ; I=1,2,3 ; p=1,2,3$ and $\mu=0,1,2,3$ refer to the $\mathrm{SU}(3), \mathrm{SU}(2)$, flavour and Lorentz indices respectively.

Research Award) and SERB/PHY/2019501 (MATRICS) and Initiation Research Grant, agreement number IITK/PHY/2015077, by IIT Kanpur. SP is supported by the MHRD, Government of India, under the Prime Minister's Research Fellows (PMRF) Scheme, 2020. M.S. is supported by the STFC under grant ST/P001246/1.

\section{A The Standard model field content}

The Standard Model degrees of freedom and their quantum numbers under space-time and internal symmetry groups have been listed in table 26 .

\section{B Products of lower dimensional SU $(N)$ representations}

Each vertex involving the light SM degrees of freedom and the candidate heavy fields must be invariant under Lorentz as well as the SM internal symmetry $\mathrm{SU}(3)_{C} \times \mathrm{SU}(2)_{L} \times \mathrm{U}(1)_{Y}$. We have already indicated how Lorentz invariance serves as a guiding principle to obtain the different categories of renormalizable vertices composed of scalars, spinors and vector bosons. The invariance with respect to the SM gauge group implies that each vertex must be an overall singlet with respect to both $\mathrm{SU}(3)$ and $\mathrm{SU}(2)$ and must posses zero $\mathrm{U}(1)$ charge. While the case of $\mathrm{U}(1)$ can be easily understood through the addition of the charges, we have discussed how invariants are constructed for the other two groups below: 
SU(2): the SM fields belong to either the singlet, the fundamental or the adjoint, i.e., the 1-, 2- or 3-dimensional representations of SU(2). The tensor products of these lower dimensional representations (elucidated by means of Young diagrams) that are useful for obtaining the results of tables $2-4$ are following [64-66]:
i.) $2 \otimes 2=3 \oplus 1$,

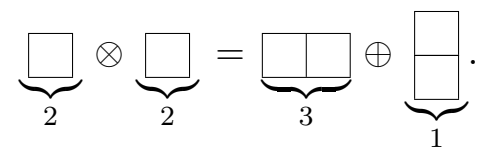

ii.) $3 \otimes 2=4 \oplus 2$,

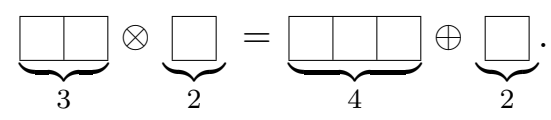

iii.) $3 \otimes 3=1 \oplus 3 \oplus 5$,

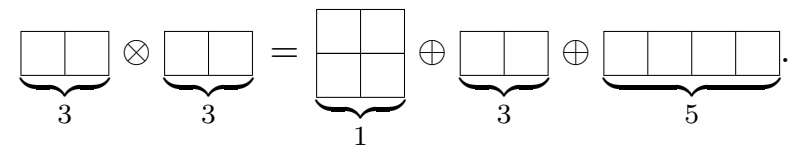

iv.) $4 \otimes 4=1 \oplus 3 \oplus 5 \oplus 7$,

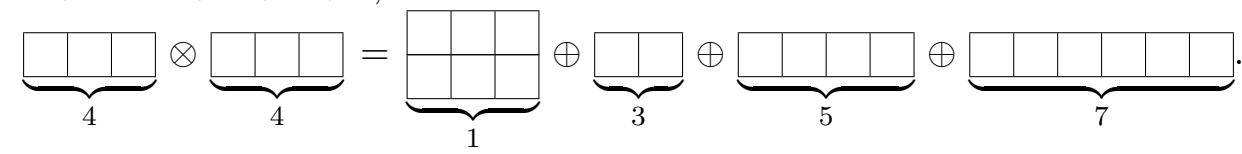

v.) In general the product of any non-trivial representation $\left(R_{L}\right)$ of $\mathrm{SU}(2)$ with itself contains both the singlet as well as the triplet (adjoint) representations: $R_{L} \otimes R_{L}=$ $1 \oplus 3 \oplus \cdots \oplus\left(2 R_{L}-1\right)$.

vi.) Similarly, some other general products which contain the doublet and triplet representations are:

$$
R_{L} \otimes\left(R_{L}-1\right)=2 \oplus 4 \oplus \cdots \oplus\left(2 R_{L}-2\right) \text { and } R_{L} \otimes\left(R_{L}-2\right)=3 \oplus 5 \oplus \cdots \oplus\left(2 R_{L}-3\right) .
$$

Based on these it can be understood why, for instance in the vertices V1-(i) and V1(ii) from table 2, which are trilinear scalar vertices with two SM Higgs ( $\mathrm{SU}(2)$ doublets), the heavy field should either be a singlet (1-dimensional) or triplet (3-dimensional) under $\mathrm{SU}(2)$, since both $2 \otimes 2 \otimes 1$ and $2 \otimes 2 \otimes 3$ can lead to an overall singlet. Similarly, in the case of the vertices V3-(i) and V3-(ii), which are quartic scalar vertices with three SM Higgs, the heavy field must either be an $\mathrm{SU}(2)$ doublet (2-dimensional) or quadruplet (4-dimensional), on account of the fact that $(2 \otimes 2) \otimes(2 \otimes 2) \supset(1 \otimes 1)=1$ or $(2 \otimes 2) \otimes(2 \otimes 2) \supset(3 \otimes 3) \supset 1$ and $(2 \otimes 2) \otimes 2 \otimes 4 \supset(3 \otimes 2) \otimes 4 \supset(4 \otimes 4) \supset 1$.

On the other hand, in the case of vertices such as V2 with one SM Higgs and two possible heavy fields, the representation cannot be fixed since we have one equation in two unknowns but we can demand that a constraint of the form $R_{L_{1}} \otimes R_{L_{2}} \supset 2$ be satisfied. Here, $R_{C_{1,2}}$ are the $\mathrm{SU}(2)$ representations of the two heavy fields. As for V9-(ii) and similar vertices containing the $\mathrm{SU}(2)_{L}$ gauge boson (which transforms as the adjoint representation), the unknown heavy field can belong to any non-trivial representation.

SU(3): the SM fields belong to either the singlet (1), fundamental (3), anti-fundamental $(\overline{3})$ or the adjoint (8) representations of $\mathrm{SU}(3)$. The relevant tensor products have been enumerated below [64-66]: 
i.) $3 \otimes \overline{3}=8 \oplus 1$,

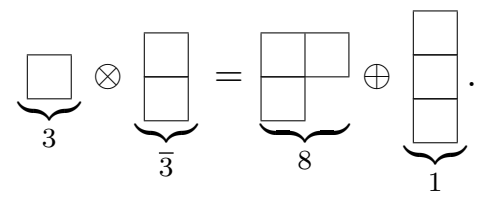

ii.) $3 \otimes 3=6 \oplus \overline{3}$,

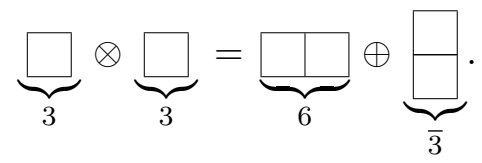

iii.) $6 \otimes 3=10 \oplus 8$,

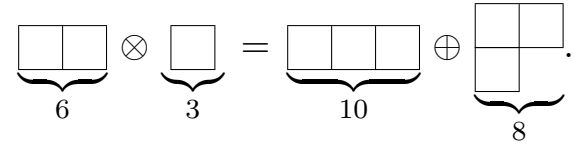

iv.) $6 \otimes \overline{3}=15 \oplus 3$,

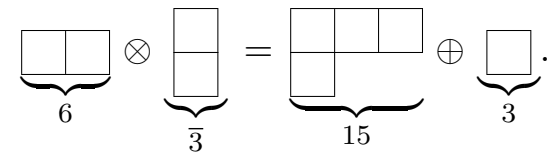

v.) $6 \otimes \overline{6}=1 \oplus 8 \oplus 27$,

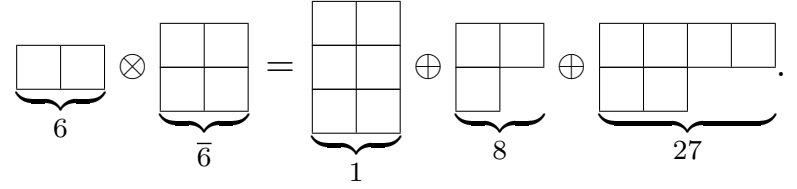

vi.) $8 \otimes 8=1 \oplus 8 \oplus 8 \oplus 10 \oplus \overline{10} \oplus 27$,

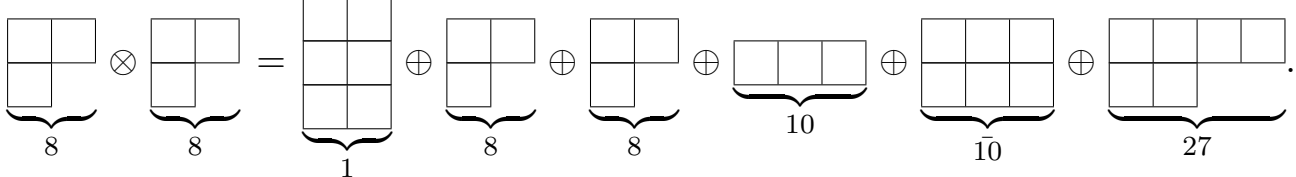

vii.) In general the product of any non-trivial representation $\left(R_{C}\right)$ of $\mathrm{SU}(3)$ and its conjugate contains both the singlet as well as the adjoint representations: $R_{C} \otimes \bar{R}_{C}=$ $1 \oplus 8 \oplus \cdots$.

Only the SM quarks and the gluons are non-trivially charged under SU(3). The vertices involving these fields and the choice of heavy field representations belong to the following categories:

- For the Yukawa-like vertices, e.g., V6-(iii)-V6-(v) from table 3 involving a quark (an $\mathrm{SU}(3)$ triplet) and the SM Higgs (an SU(3) singlet), the heavy fermion can only be a $\overline{3}$ under $\mathrm{SU}(3)$. The same also holds for the vertices V6-(viii)-V6-(x)

- For vertices such as V5-(iii)-V5-(v) and V5-(x) involving 2 quarks, the heavy scalar can either be a 3 or $\overline{6}$ under $\mathrm{SU}(3)$, on account of the fact that $(3 \otimes 3) \otimes 3 \supset(\overline{3} \otimes 3) \supset 1$ and $(3 \otimes 3) \otimes \overline{6} \supset(6 \otimes \overline{6}) \supset 1$.

- Similarly, for vertices such as V5-(vii) and V5-(viii), involving a quark and an antiquark, the heavy scalar can only be a singlet (1) or octet (8) under SU(3).

- The vertices V7-(iii)-V7-(v), on the other hand, contain only one SM quark and requires a heavy scalar as well as a heavy fermion. In this case, we cannot fix the exact representations to which these fields belong but we can impose a constraint that in each case $R_{C_{1}} \otimes R_{C_{2}} \supset \overline{3}$ must hold in each case, where $R_{C_{1,2}}$ are the $\mathrm{SU}(3)$ representations of the two heavy fields. 
- For V9-(iii) and similar vertices containing the SU(3) gauge bosons, i.e., gluons which transforms as the adjoint representation, the unknown heavy field can belong to any non-trivial representation of $\mathrm{SU}(3)$.

Open Access. This article is distributed under the terms of the Creative Commons Attribution License (CC-BY 4.0), which permits any use, distribution and reproduction in any medium, provided the original author(s) and source are credited.

\section{References}

[1] S. Weinberg, Effective Gauge Theories, Phys. Lett. B 91 (1980) 51 [InSPIRE].

[2] W. Buchmüller and D. Wyler, Effective Lagrangian Analysis of New Interactions and Flavor Conservation, Nucl. Phys. B 268 (1986) 621 [INSPIRE].

[3] B. Grzadkowski, M. Iskrzynski, M. Misiak and J. Rosiek, Dimension-Six Terms in the Standard Model Lagrangian, JHEP 10 (2010) 085 [arXiv: 1008.4884] [INSPIRE].

[4] J.C. Criado, BasisGen: automatic generation of operator bases, Eur. Phys. J. C 79 (2019) 256 [arXiv: 1901.03501$]$ [INSPIRE].

[5] R.M. Fonseca, The Sym2Int program: going from symmetries to interactions, J. Phys. Conf. Ser. 873 (2017) 012045 [arXiv: 1703.05221] [INSPIRE].

[6] B. Gripaios and D. Sutherland, DEFT: A program for operators in EFT, JHEP 01 (2019) 128 [arXiv: 1807.07546] [INSPIRE].

[7] U. Banerjee, J. Chakrabortty, S. Prakash and S.U. Rahaman, Characters and group invariant polynomials of (super)fields: road to "Lagrangian", Eur. Phys. J. C 80 (2020) 938 [arXiv: 2004.12830] [INSPIRE].

[8] C.B. Marinissen, R. Rahn and W.J. Waalewijn, ..., 83106786, 114382724, 1509048322, 2343463290, 27410087742, . . efficient Hilbert series for effective theories, Phys. Lett. B 808 (2020) 135632 [arXiv:2004.09521] [INSPIRE].

[9] S. Weinberg, Phenomenological Lagrangians, Physica A 96 (1979) 327 [InSPIRE].

[10] B. Henning, X. Lu, T. Melia and H. Murayama, 2, 84, 30, 993, 560, 15456, 11962, 261485 , ...: Higher dimension operators in the SM EFT, JHEP 08 (2017) 016 [Erratum JHEP 09 (2019) 019] [arXiv: 1512.03433] [INSPIRE].

[11] L. Lehman, Extending the Standard Model Effective Field Theory with the Complete Set of Dimension-7 Operators, Phys. Rev. D 90 (2014) 125023 [arXiv:1410.4193] [INSPIRE].

[12] C.W. Murphy, Dimension-8 operators in the Standard Model Eective Field Theory, JHEP 10 (2020) 174 [arXiv : 2005. 00059] [inSPIRE].

[13] H.-L. Li, Z. Ren, J. Shu, M.-L. Xiao, J.-H. Yu and Y.-H. Zheng, Complete Set of Dimension-8 Operators in the Standard Model Effective Field Theory, arXiv:2005.00008 [INSPIRE].

[14] H.-L. Li, Z. Ren, M.-L. Xiao, J.-H. Yu and Y.-H. Zheng, Complete Set of Dimension-9 Operators in the Standard Model Effective Field Theory, arXiv:2007.07899 [InSPIRE].

[15] Y. Liao and X.-D. Ma, An explicit construction of the dimension-9 operator basis in the standard model effective field theory, JHEP 11 (2020) 152 [arXiv:2007.08125] [INSPIRE].

[16] H. Georgi, Effective field theory, Ann. Rev. Nucl. Part. Sci. 43 (1993) 209 [InSPIRE]. 
[17] B. Henning, X. Lu and H. Murayama, How to use the Standard Model effective field theory, JHEP 01 (2016) 023 [arXiv: 1412.1837] [INSPIRE].

[18] B. Henning, X. Lu and H. Murayama, One-loop Matching and Running with Covariant Derivative Expansion, JHEP 01 (2018) 123 [arXiv:1604.01019] [INSPIRE].

[19] U. Haisch, M. Ruhdorfer, E. Salvioni, E. Venturini and A. Weiler, Singlet night in Feynman-ville: one-loop matching of a real scalar, JHEP 04 (2020) 164 [Erratum JHEP 07 (2020) 066] [arXiv:2003.05936] [INSPIRE].

[20] M. Jiang, N. Craig, Y.-Y. Li and D. Sutherland, Complete one-loop matching for a singlet scalar in the Standard Model EFT, JHEP 02 (2019) 031 [Erratum JHEP 01 (2021) 135] [arXiv: 1811.08878] [INSPIRE].

[21] Z. Zhang, Covariant diagrams for one-loop matching, JHEP 05 (2017) 152 [arXiv: 1610.00710] [INSPIRE].

[22] S.A.R. Ellis, J. Quevillon, T. You and Z. Zhang, Mixed heavy-light matching in the Universal One-Loop Effective Action, Phys. Lett. B 762 (2016) 166 [arXiv:1604.02445] [INSPIRE].

[23] S.A.R. Ellis, J. Quevillon, T. You and Z. Zhang, Extending the Universal One-Loop Effective Action: Heavy-Light Coefficients, JHEP 08 (2017) 054 [arXiv: 1706. 07765] [INSPIRE].

[24] S.A.R. Ellis, J. Quevillon, P.N.H. Vuong, T. You and Z. Zhang, The Fermionic Universal One-Loop Effective Action, JHEP 11 (2020) 078 [arXiv:2006.16260] [INSPIRE].

[25] F. del Aguila, Z. Kunszt and J. Santiago, One-loop effective lagrangians after matching, Eur. Phys. J. C 76 (2016) 244 [arXiv: 1602.00126] [INSPIRE].

[26] M. Krämer, B. Summ and A. Voigt, Completing the scalar and fermionic Universal One-Loop Effective Action, JHEP 01 (2020) 079 [arXiv: 1908.04798] [INSPIRE].

[27] A. Drozd, J. Ellis, J. Quevillon and T. You, The Universal One-Loop Effective Action, JHEP 03 (2016) 180 [arXiv: 1512.03003] [INSPIRE].

[28] S. Das Bakshi, J. Chakrabortty and S.K. Patra, CoDEx: Wilson coefficient calculator connecting SMEFT to UV theory, Eur. Phys. J. C 79 (2019) 21 [arXiv: 1808.04403] [INSPIRE].

[29] J. Aebischer, J. Kumar and D.M. Straub, Wilson: a Python package for the running and matching of Wilson coefficients above and below the electroweak scale, Eur. Phys. J. C 78 (2018) 1026 [arXiv: 1804.05033] [INSPIRE].

[30] J.C. Criado, MatchingTools: a Python library for symbolic effective field theory calculations, Comput. Phys. Commun. 227 (2018) 42 [arXiv:1710.06445] [INSPIRE].

[31] A. Celis, J. Fuentes-Martin, A. Vicente and J. Virto, DsixTools: The Standard Model Effective Field Theory Toolkit, Eur. Phys. J. C 77 (2017) 405 [arXiv:1704.04504] [INSPIRE].

[32] N.G. Deshpande and E. Ma, Pattern of Symmetry Breaking with Two Higgs Doublets, Phys. Rev. D 18 (1978) 2574 [INSPIRE].

[33] G. Bambhaniya, J. Chakrabortty, S. Goswami and P. Konar, Generation of neutrino mass from new physics at TeV scale and multilepton signatures at the LHC, Phys. Rev. D 88 (2013) 075006 [arXiv:1305.2795] [INSPIRE].

[34] J. de Blas, M. Chala, M. Pérez-Victoria and J. Santiago, Observable Effects of General New Scalar Particles, JHEP 04 (2015) 078 [arXiv: 1412.8480] [INSPIRE]. 
[35] A. Arhrib et al., The Higgs Potential in the Type II Seesaw Model, Phys. Rev. D 84 (2011) 095005 [arXiv: 1105.1925] [INSPIRE].

[36] A. Angelescu and P. Huang, Integrating Out New Fermions at One Loop, JHEP 01 (2021) 049 [arXiv: 2006.16532] [inSPIRE].

[37] P. Duka, J. Gluza and M. Zralek, Quantization and renormalization of the manifest left-right symmetric model of electroweak interactions, Annals Phys. 280 (2000) 336 [hep-ph/9910279] [INSPIRE].

[38] N.G. Deshpande, J.F. Gunion, B. Kayser and F.I. Olness, Left-right symmetric electroweak models with triplet Higgs, Phys. Rev. D 44 (1991) 837 [InSPIRE].

[39] S. Saad, Fermion Masses and Mixings, Leptogenesis and Baryon Number Violation in Pati-Salam Model, Nucl. Phys. B 943 (2019) 114630 [arXiv:1712.04880] [InSPIRE].

[40] J. de Blas, J.C. Criado, M. Pérez-Victoria and J. Santiago, Effective description of general extensions of the Standard Model: the complete tree-level dictionary, JHEP 03 (2018) 109 [arXiv: 1711.10391] [INSPIRE].

[41] S. Das Bakshi, J. Chakrabortty and M. Spannowsky, Classifying Standard Model Extensions Effectively with Precision Observables, Phys. Rev. D 103 (2021) 056019 [arXiv: 2012.03839] [INSPIRE].

[42] V. Gherardi, D. Marzocca and E. Venturini, Matching scalar leptoquarks to the SMEFT at one loop, JHEP 07 (2020) 225 [Erratum JHEP 01 (2021) 006] [arXiv: 2003.12525] [INSPIRE].

[43] S. Dawson, S. Homiller and S.D. Lane, Putting standard model EFT fits to work, Phys. Rev. D 102 (2020) 055012 [arXiv: 2007.01296] [INSPIRE].

[44] J. Ellis, C.W. Murphy, V. Sanz and T. You, Updated Global SMEFT Fit to Higgs, Diboson and Electroweak Data, JHEP 06 (2018) 146 [arXiv:1803.03252] [INSPIRE].

[45] J. Gargalionis and R.R. Volkas, Exploding operators for Majorana neutrino masses and beyond, JHEP 01 (2021) 074 [arXiv:2009.13537] [INSPIRE].

[46] I. Brivio, Y. Jiang and M. Trott, The SMEFTsim package, theory and tools, JHEP 12 (2017) 070 [arXiv: 1709.06492] [INSPIRE].

[47] I. Brivio and M. Trott, The Standard Model as an Effective Field Theory, Phys. Rept. 793 (2019) 1 [arXiv: 1706. 08945] [INSPIRE].

[48] S. Dawson and P.P. Giardino, Electroweak and QCD corrections to $Z$ and $W$ pole observables in the standard model EFT, Phys. Rev. D 101 (2020) 013001 [arXiv:1909.02000] [INSPIRE].

[49] J. Baglio, S. Dawson, S. Homiller, S.D. Lane and I.M. Lewis, Validity of standard model EFT studies of VH and VV production at NLO, Phys. Rev. D 101 (2020) 115004 [arXiv: 2003. 07862] [INSPIRE].

[50] S. Das Bakshi, J. Chakrabortty, C. Englert, M. Spannowsky and P. Stylianou, CP violation at ATLAS in effective field theory, Phys. Rev. D 103 (2021) 055008 [arXiv:2009.13394] [INSPIRE].

[51] Anisha, S. Das Bakshi, J. Chakrabortty and S.K. Patra, Connecting electroweak-scale observables to BSM physics through EFT and Bayesian statistics, Phys. Rev. D 103 (2021) 076007 [arXiv: 2010.04088] [INSPIRE]. 
[52] M.K. Gaillard, The Effective One Loop Lagrangian With Derivative Couplings, Nucl. Phys. B 268 (1986) 669 [INSPIRE].

[53] O. Cheyette, Derivative Expansion of the Effective Action, Phys. Rev. Lett. 55 (1985) 2394 [INSPIRE].

[54] M.S. Bilenky and A. Santamaria, One loop effective Lagrangian for a standard model with a heavy charged scalar singlet, Nucl. Phys. B 420 (1994) 47 [hep-ph/9310302] [INSPIRE].

[55] A.V. Manohar, Introduction to Effective Field Theories, in Lecture notes of the Les Houches Summer School 108, Oxford University Press, Oxford U.K. (2020) [arXiv: 1804.05863] [INSPIRE].

[56] L.H. Chan, Effective action expansion in perturbation theory, Phys. Rev. Lett. 54 (1985) 1222 [Erratum ibid. 56 (1986) 404] [INSPIRE].

[57] U. Banerjee, J. Chakrabortty, S. Prakash, S.U. Rahaman and M. Spannowsky, Effective Operator Bases for Beyond Standard Model Scenarios: An EFT compendium for discoveries, JHEP 01 (2021) 028 [arXiv:2008.11512] [INSPIRE].

[58] G.C. Branco, P.M. Ferreira, L. Lavoura, M.N. Rebelo, M. Sher and J.P. Silva, Theory and phenomenology of two-Higgs-doublet models, Phys. Rept. 516 (2012) 1 [arXiv:1106.0034] [INSPIRE].

[59] G. Bhattacharyya and D. Das, Scalar sector of two-Higgs-doublet models: A minireview, Pramana 87 (2016) 40 [arXiv:1507.06424] [INSPIRE].

[60] E.E. Jenkins, A.V. Manohar and M. Trott, Renormalization Group Evolution of the Standard Model Dimension Six Operators I: Formalism and lambda Dependence, JHEP 10 (2013) 087 [arXiv: 1308.2627] [INSPIRE].

[61] Anisha, S. Das Bakshi, J. Chakrabortty and S. Prakash, Hilbert Series and Plethystics: Paving the path towards 2HDM- and MLRSM-EFT, JHEP 09 (2019) 035 [arXiv: 1905.11047] [INSPIRE].

[62] A. Crivellin, M. Ghezzi and M. Procura, Effective Field Theory with Two Higgs Doublets, JHEP 09 (2016) 160 [arXiv:1608.00975] [INSPIRE].

[63] S. Karmakar and S. Rakshit, Relaxed constraints on the heavy scalar masses in 2HDM, Phys. Rev. D 100 (2019) 055016 [arXiv:1901.11361] [InSPIRE].

[64] R. Slansky, Group Theory for Unified Model Building, Phys. Rept. 79 (1981) 1 [inSPIRE].

[65] W. Fulton, Young Tableaux: With Applications to Representation Theory and Geometry, in London Mathematical Society Student Texts, Cambridge University Press, Cambridge U.K. (1996).

[66] R. Feger, T.W. Kephart and R.J. Saskowski, LieART 2.0 - A Mathematica application for Lie Algebras and Representation Theory, Comput. Phys. Commun. 257 (2020) 107490 [arXiv: 1912.10969] [INSPIRE]. 